TOPICAL REVIEW

\title{
Expectations at LHC from hard QCD
}

\author{
S Moch \\ Deutsches Elektronensynchrotron DESY, Platanenallee 6, D-15738 Zeuthen, Germany
}

Received 4 March 2008

Published 14 May 2008

Online at stacks.iop.org/JPhysG/35/073001

\begin{abstract}
We review the status of QCD at hadron colliders with emphasis on precision predictions and the latest theoretical developments for cross-section calculations to higher orders. We include an overview of our current information on parton distributions and discuss various Standard Model reactions such as $W^{ \pm} / Z$-boson, Higgs boson or top quark production.
\end{abstract}

\section{Introduction}

Historically, hadron colliders have explored elementary particle physics at the energy frontier, the motivation being the discovery of new particles through direct production. This has been the case for the SppS at CERN leading to the discovery of the weak vector bosons as well as for Tevatron at Fermilab currently operating at a center-of-mass energy $\sqrt{S}=1.96 \mathrm{TeV}$ with the discovery of the top quark. Shortly the Large Hadron Collider (LHC) at CERN with $\sqrt{S}=14 \mathrm{TeV}$ will commence operation which will realize a major leap forward in collision energy. Being long awaited the machine will allow access to the mechanism of electroweak symmetry breaking, to search for the Higgs boson and, hopefully, it will open new avenues to test many proposed extensions of the Standard Model. To that end, two general purpose experiments ATLAS and CMS [1-4] as well as two specialized ones, LHCb for B-physics [5] and Alice for heavy-ion physics [6], have been installed.

The expected cross sections for proton-proton scattering at LHC is large (see figure 1). In particular we will have large rates for many Standard Model processes such as the production of $b$-quarks, $W^{ \pm}$- and $Z$-bosons, jets (even with high cuts on the transverse momentum) and top quarks. Much of the physics is actually dominated by the gauge theory of the strong interactions, quantum chromodynamics (QCD). Comparing the rates for various processes at Tevatron and LHC in figure 1, it is obvious that any search for physics beyond the Standard Model (BSM) like for superpartners in supersymmetric extensions (squarks, gluinos, ...), for Kaluza-Klein modes in models with extra dimensions or even the search for the Higgs boson needs a very precise understanding of the known background from the Standard Model. Thus, new physics searches require precision predictions, most importantly in QCD. Moreover, the era of LHC implies a change of paradigm. We no longer test QCD, rather we use perturbative QCD as an essential and established part of our theory toolkit. 


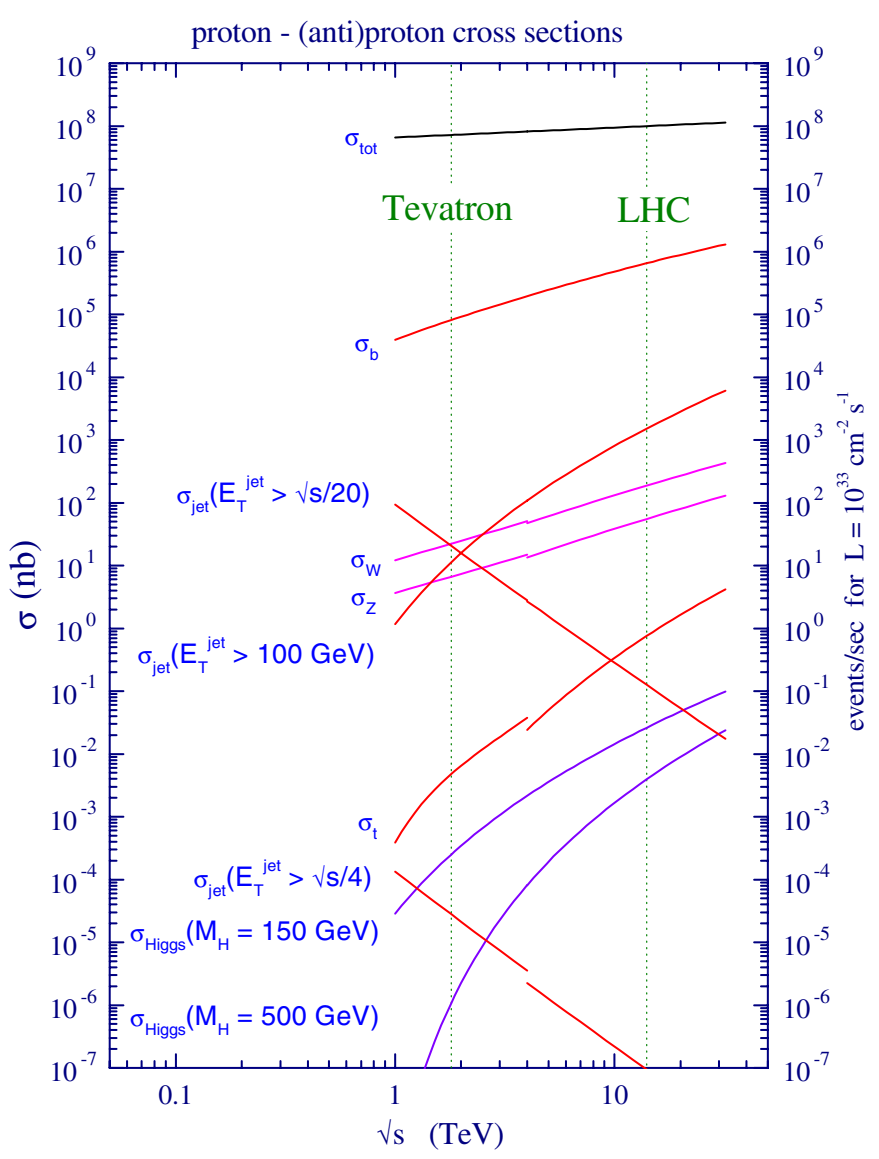

Figure 1. Predictions for hard-scattering cross sections in $p \bar{p}$ collision at Tevatron and in $p p$ collision at LHC as a function of the center-of-mass energy (Reprinted from Rep. Prog. Phys. 7089 with permission from IOP Publishing).

Hard QCD is a large subject and, necessarily, the coverage here has to be selective (see $[7,8]$ for other recent reviews on this topic). In this review, we will briefly review the physics concepts and theoretical framework for hard-scattering reactions at hadron colliders, focusing on QCD. We point out achievements of the past years as well as open problems. We will briefly explain the property of factorization and discuss the parton luminosity in proton collisions. We will summarize the present knowledge on hard parton scattering cross sections for the production of $W^{ \pm}$and $Z$ gauge bosons, jets in QCD, heavy quarks, such as top and bottom and the Higgs. We put particular emphasis on exact calculations of radiative corrections in QCD to next-to-leading order (NLO), next-to-next-to-leading order (NNLO) or beyond. Many other aspects such as, e.g., the Monte Carlo approach to modeling hadronic interactions and parton showers we can only touch briefly or else have to refer to the literature.

With many Standard Model processes to be measured in the early days of LHC and the associated uncertainties to be understood on the way to the discovery of new physics [9], we hope that this review serves to illustrate a few aspects common to the underlying QCD dynamics. 


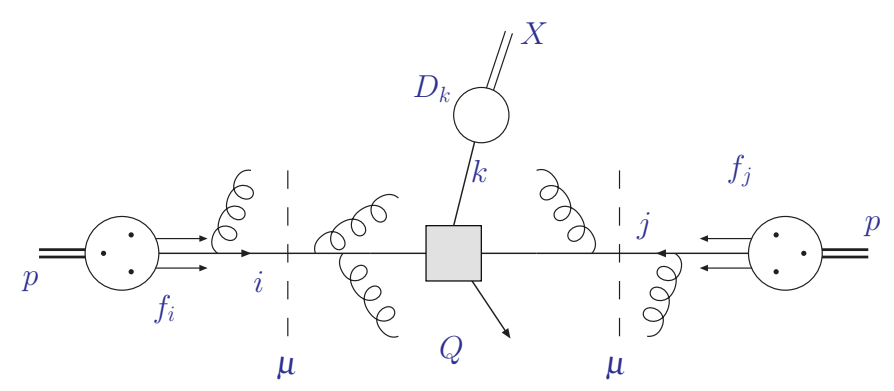

Figure 2. Factorization for the hard-scattering cross sections in equation (1) in the QCD-improved parton model.

\section{Perturbative QCD at colliders}

The basic prerequisite for the application of perturbative QCD at colliders is factorization for hard-scattering processes. For hard hadron-hadron scattering this property implies that the constituent partons from each incoming hadron interact at short distance (i.e. at large momentum transfer $Q^{2}$ ). The property of $\mathrm{QCD}$ factorization rests on the fact that we can separate the sensitivity to dynamics from different scales (see, e.g., [10]). Thus, for a cross section $\sigma_{p p \rightarrow X}$ of some hadronic final state $X$ in, say, proton-proton scattering we can write

$$
\begin{aligned}
\sigma_{p p \rightarrow X}=\sum_{i j k} \int & \mathrm{d} x_{1} \mathrm{~d} x_{2} \mathrm{~d} z f_{i}\left(x_{1}, \mu^{2}\right) f_{j}\left(x_{2}, \mu^{2}\right) \\
& \times \hat{\sigma}_{i j \rightarrow k}\left(x_{1}, x_{2}, z, Q^{2}, \alpha_{s}\left(\mu^{2}\right), \mu^{2}\right) D_{k \rightarrow X}\left(z, \mu^{2}\right),
\end{aligned}
$$

where all functions have a clear physical interpretation.

The parton distribution functions (PDFs) in the proton $f_{i}(i=q, \bar{q}, g)$ describe the fraction $x_{i}$ of the hadron momentum carried by the quark or gluon and the convolution of $f_{i}$ and $f_{j}$ determines the parton luminosity at the collider. The PDFs cannot be calculated in perturbation theory due to the proton being a very complicated multi-particle bound state. Rather, they have to be obtained from global fits to experimental data. The (hard) parton cross section $\hat{\sigma}_{i j \rightarrow k}$ depending on the parton types $i, j$ and $k$ is calculable perturbatively in QCD in powers of the strong coupling constant $\alpha_{s}$ and describes how the constituent partons from incoming protons interact at short distances of order $\mathcal{O}(1 / Q)$. The final state $X$ may denote hadrons, mesons, jets, etc and needs another transition from the perturbative hard partons in the final state to the observed particles. The necessary function $D_{k \rightarrow X}$ can therefore be a fragmentation function or also a jet algorithm. Here, the interface with showering algorithms (based on a Monte Carlo approach) becomes particularly crucial. All quantities in equation (1) depend on the renormalization and factorization scale, $\mu_{r}$ and $\mu_{f}$, which are usually taken to be the same. Throughout this review we set $\mu_{r}=\mu_{f}=\mu$. The details of the integration range in the convolution in equation (1) are controlled by the kinematics of the hard-scattering process. Schematically, QCD factorization can be depicted as in figure 2 .

Physical observables like the cross section $\sigma_{p p \rightarrow X}$ in equation (1) cannot depend on the factorization scale. In the perturbative approach, this implies that any dependence on $\mu$ in $\sigma_{p p \rightarrow X}$ has to vanish at least to the order in $\alpha_{s}$ considered. This property can be cast in the following form:

$$
\frac{\mathrm{d}}{\mathrm{d} \ln \mu^{2}} \sigma_{p p \rightarrow X}=\mathcal{O}\left(\alpha_{s}^{l+1}\right)
$$


It defines the commonly adopted approach to quantify uncertainties in theoretical predictions based on the variation of the renormalization and factorization scale.

Let us briefly turn to hard-scattering cross sections. There exist various approaches to the calculation of $\sigma_{p p \rightarrow X}$ ranging from easy to difficult as far as computational complexity is concerned as well as from inclusive to fully differential in terms of kinematical variables. First of all, there exist parton shower Monte Carlos (e.g. Herwig [11], Pythia [12, 13], Sherpa [14]) which are very important tools for understanding multi-parton scattering and the underlying event.

For predictions building on exact matrix elements at leading order (LO), we have at our disposal many automated tree level calculations in the Standard Model, in its minimal supersymmetric extension (MSSM) or in other BSM models utilizing programs such as, e.g., Alpgen [15], CompHEP [16], Helac-Phegas [17], MadGraph [18] or Whizard [19]. These tools provide first estimates for hard-scattering cross sections through numerical phase-space integration of the exact matrix elements. In this way, they are flexible as far as kinematics and the topology of a given hard-scattering observable is concerned and allow easy interfacing of LO calculations with parton shower Monte Carlos and, possibly, detector simulation. However, scattering reactions with exact matrix elements for more than eight jets (particles) in the final state are currently at the edge of computational capabilities.

At NLO level we do have some analytical (or numerical) calculations of Feynman diagrams yielding parton level Monte Carlos (e.g. NLOJET++ [20, 21] or MCFM [22]). However, we have also seen recently significant progress based on string inspired techniques. At the edge of technical developments is the concept of exact NLO calculations interfaced with parton shower in programs as realized in MC@NLO [23, 24], POWHEG [25, 26] or VINCIA [27].

At higher orders in QCD perturbation theory, like NNLO, some selected results are known mostly for inclusive kinematics. However, in view of LHC, we have witnessed significant progress in the last years to also provide predictions in completely differential kinematics [28, 29]. Beyond this level of accuracy at, say, next-to-next-to-next-to-leading order $\left(\mathrm{N}^{3} \mathrm{LO}\right)$ only very few results are known, e.g. for deep-inelastic scattering (DIS) [30].

\section{Parton luminosity at hadron colliders}

The parton luminosity in equation (1) is an indispensable ingredient of hard-scattering processes involving initial-state hadrons. At hadron colliders one has wide-band beams of quarks and gluons and, as is well known, the necessary PDFs of the proton $f_{i}(i=q, \bar{q}, g)$ are not directly accessible in QCD perturbation theory. However, the scale dependence (evolution) of PDFs is governed by the splitting functions and predicted in a perturbative expansion in powers of $\alpha_{s}$. The universality allows for the determination of sets of PDFs in global fits to experimental data. Upon evolution this information from fits to reference processes can be used to provide cross-section predictions at LHC energies and we can quantify the present uncertainties.

\subsection{Parton evolution}

The parton distributions in the hadron are distinguished by the flavor quantum numbers, which are additive. The valence distribution originates from differences of quarks and anti-quarks $q-\bar{q}$. The proton is composed of the sea distribution (i.e. the sum over all flavors $q+\bar{q}$ ) and of the gluon $g$. 

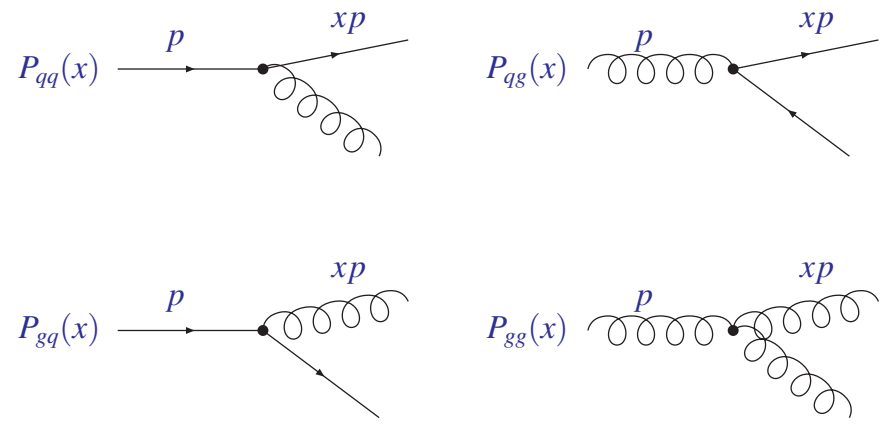

Figure 3. Sample of Feynman diagrams for parton-parton splitting in leading-order QCD. We indicate the collinear momentum flow ( $p$ incoming and $x p$ outgoing) as it enters the calculation of the corresponding splitting function $P_{i j}$. See, e.g., [31] for an operator definition of parton distributions.

Table 1. The number of Feynman diagrams contributing to parton $(q, g)$-boson DIS (vector bosons $\gamma / W$ or scalar $\phi)$ up to three loops. The NNLO splitting functions $P_{i j}$ have been determined from the collinear singularity of these scattering reactions (see $[32,33])$.

\begin{tabular}{llccc}
\hline & Tree & 1-Loop & 2-Loop & 3-Loop \\
\hline$q \gamma$ & 1 & 3 & 25 & 359 \\
$g \gamma$ & & 2 & 17 & 345 \\
$q W$ & 1 & 3 & 32 & 589 \\
$q \phi$ & & 1 & 23 & 696 \\
$g \phi$ & 1 & 8 & 218 & 6378 \\
Sum & 3 & 17 & 315 & 8367 \\
\hline
\end{tabular}

The independence of any physical observable on the scale $\mu$ immediately gives rise to evolution equations for the PDFs $f_{i}, i=q, \bar{q}, g$. From equation (2) we find that the scale dependence of $f_{i}$ is governed by

$\frac{\mathrm{d}}{\mathrm{d} \ln \mu^{2}}\left(\begin{array}{c}f_{q_{i}}\left(x, \mu^{2}\right) \\ f_{g}\left(x, \mu^{2}\right)\end{array}\right)=\sum_{j} \int_{x}^{1} \frac{\mathrm{d} z}{z}\left(\begin{array}{cc}P_{q_{i} q_{j}}(z) & P_{q_{i} g}(z) \\ P_{g q_{j}}(z) & P_{g g}(z)\end{array}\right)\left(\begin{array}{l}f_{q_{j}}\left(x / z, \mu^{2}\right) \\ f_{g}\left(x / z, \mu^{2}\right)\end{array}\right)$,

which is a system of coupled integro-differential equations corresponding to the different possible parton splittings, see e.g. figure 3 where some Feynman diagrams contributing in leading-order QCD are displayed. The splitting functions $P_{i j}$, i.e., the kernels of these differential equations are universal quantities and can be calculated in perturbation theory from the collinear singularity of any hard-scattering process. Thus, $P$ has an expansion in powers of $\alpha_{s}$ as

$$
P=\alpha_{s} P^{(0)}+\alpha_{s}^{2} P^{(1)}+\alpha_{s}^{3} P^{(2)}+\cdots,
$$

where we have suppressed parton indices. The first two terms are needed for NLO predictions, which is the standard approximation, although often still with large uncertainties. Currently, the splitting functions are known to NNLO and in table 1 we give the number of Feynman diagrams for the corresponding hard parton reactions in DIS from which the NNLO expressions $P^{(2)}$ in equation (4) have been calculated $[32,33]$. 

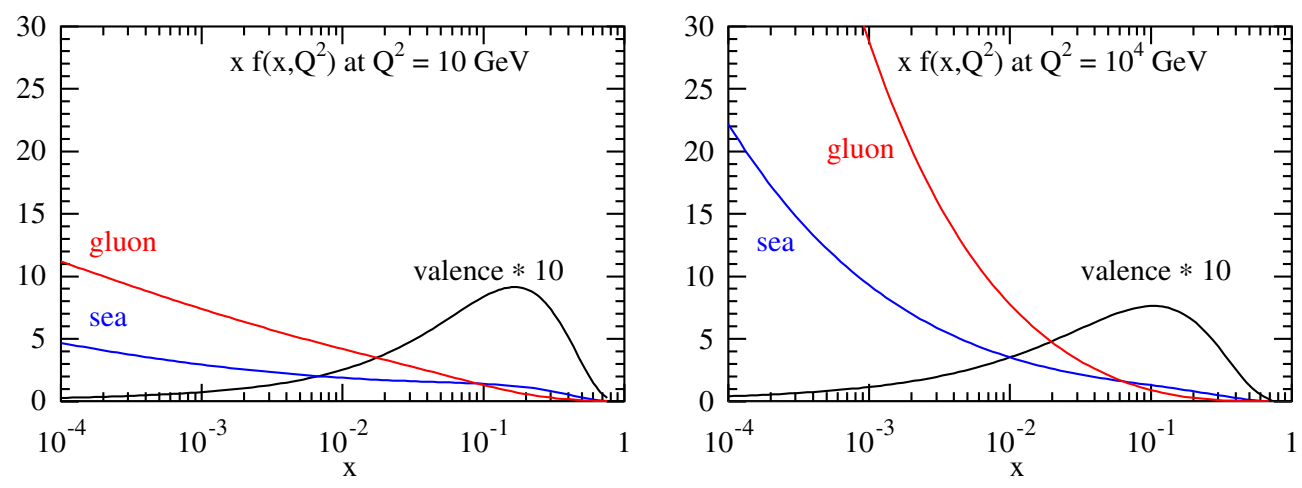

Figure 4. Evolution of the valence, sea and gluon momentum distributions $x f\left(x, Q^{2}\right)$ in the proton from a low scale at $Q^{2}=10 \mathrm{GeV}$ (left) to LHC energies at $Q^{2}=10^{4} \mathrm{GeV}$ (right) for the parameterization of [39] showing the strong rise of the gluon at small $x$.

Physically, the evolution equation (3) states that one becomes sensitive to lower momentum partons as the resolution of the proton is increased, i.e. as the scale $\mu$ becomes larger. Given an input distribution at a low scale, say $Q^{2}=10 \mathrm{GeV}^{2}$, which has to be determined in a global fit from comparison to data, one can solve equation (3) to predict the PDFs at a high scale (see figure 4). Solutions of equation (3) can be obtained by a variety of methods with available codes [34-36] and benchmarks are provided in [37, 38].

Modern parameterizations of parton distribution from global fits account in particular for the effects of experimental errors and come with the according uncertainties, see e.g. the framework LHAPDF accord [37, 40, 41]. Much of the needed experimental information originates from deep-inelastic scattering data on structure functions from HERA for $e^{ \pm} p$ scattering (H1, ZEUS) and from fixed targets (proton and deuterium) for $\mu p$ and $\mu d$ scattering (BCDMS, NMC, SLAC, E665) as well as (anti-)neutrino-proton scattering (CCFR), see e.g. [42]. These data determine the quark distributions for light flavors at all momentum fractions $x$ and through the scale evolution in perturbative QCD the gluon distribution at medium and small $x$. Further information on the flavor content of the nucleon is provided by structure function data for $F_{2}^{\text {charm }}$ from HERA for the charm distribution and by Drell-Yan data on proton-nucleon targets (E605, E772, E866), which determine the sea quark distributions, in particular $f_{\bar{u}}$ and $f_{\bar{d}}$. The Tevatron experiments CDF and D0 are able to constrain the ratio $f_{u} / f_{d}$ at high $x$ with the rapidity asymmetry in $W$-boson production and the gluon distribution at high $x$ with the help of inclusive jet data. More recently, by relaxing the assumption $f_{s}=f_{\bar{s}}$, also information on the strange asymmetry $f_{s}$ and $f_{\bar{s}}$ has been extracted from $v(\bar{v}) p$ scattering (NuTeV, CCFR).

\subsection{Parton distributions from HERA to LHC}

Given the great importance of deep-inelastic scattering data a question that has been frequently addressed in the past is, of course, the impact HERA data for LHC predictions, in particular as far as the parton luminosity is concerned (see [38, 43] for further discussion).

As illustrated by the compilation of measurements in figure 5 (left), the data on the structure function $F_{2}$ in deep-inelastic $e^{ \pm} p$ scattering extend over a wide range in $x$ and $Q^{2}$. Considering, on the other hand, the allowed region for parton kinematics at LHC in figure 5 (right), it is clear that there is a large overlap in $x$ with the range covered by HERA. However, 

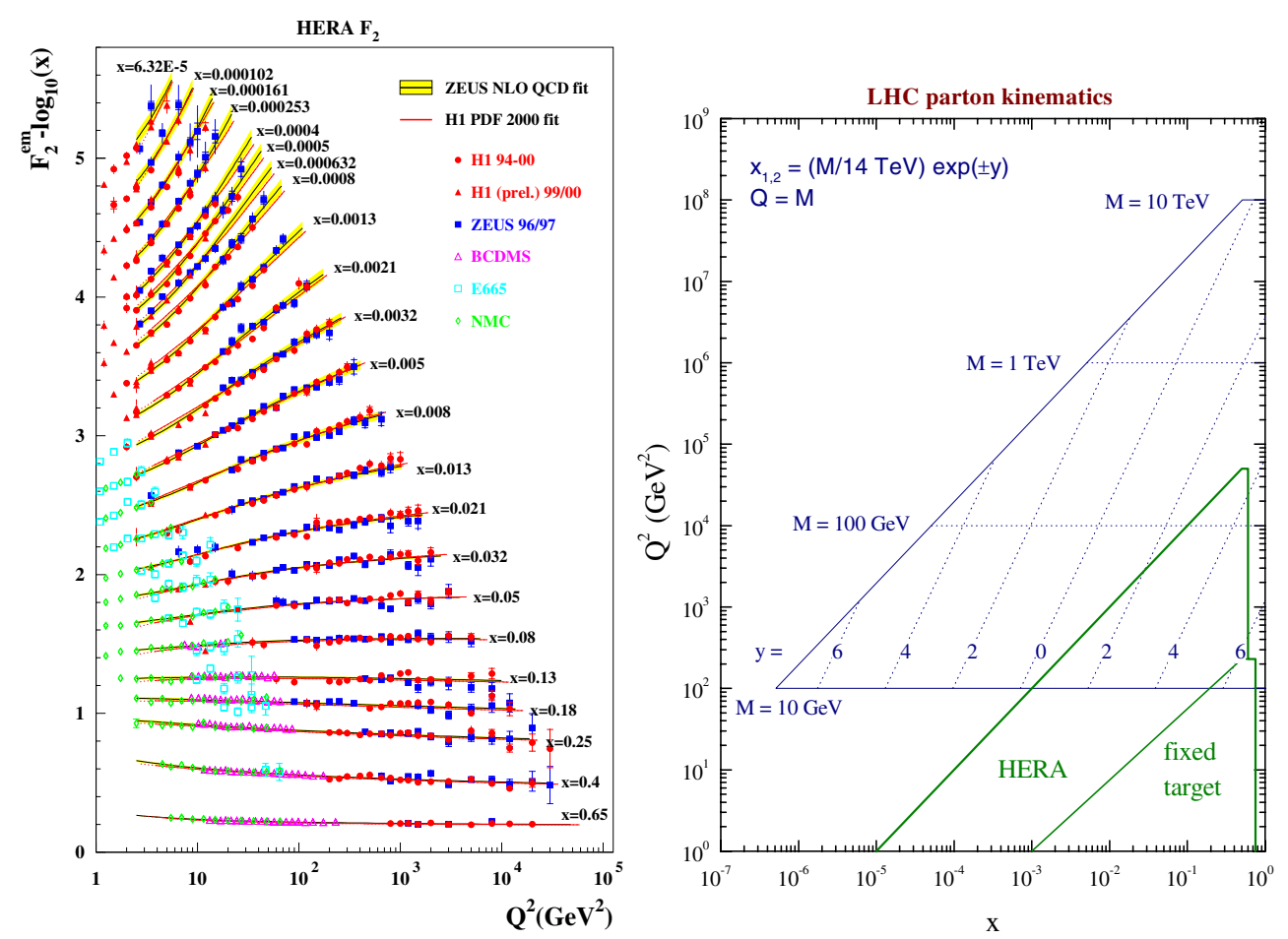

Figure 5. Left: the structure function $F_{2}$ in deep-inelastic $e^{ \pm} p$ scattering as a function of $x$ and $Q^{2}$ Reprinted with permission from 2003 Phys. Rev. D 67012007 . Copyright (2003) by the American Physical Society. Right: parton kinematics at LHC and at HERA (Reprinted from 2007 Rep. Prog. Phys. 7089 with permission from IOP Publishing).

the relevant hard scale $Q$ is typically two to three orders higher due to the increased center-ofmass energy $\sqrt{S}$. Details of the parton kinematics at LHC depend, of course, on the invariant mass $M$ of the final state and on the rapidity $y$. The dominant values of the momentum fractions are $x_{1,2} \sim\left(M e^{ \pm y}\right) / \sqrt{S}$ and variation of $M$ and $y$ at fixed $\sqrt{S}$ tests the sensitivity to partons with different momentum fractions.

The large difference in the hard momentum scale $Q$ between HERA and LHC requires the parton evolution based on equation (3) to be sufficiently accurate in perturbative QCD. The necessary perturbative accuracy for quantitative predictions is approached at NNLO $[32,33]$. The stability of evolution is shown in figure 6 , where the scale derivatives of quark and gluon distributions at $\mu^{2} \approx 30 \mathrm{GeV}^{2}$ are displayed. Obviously, the expansion is very stable except for very small momentum fractions $x \lesssim 10^{-4}$ which shows that the perturbative evolution equation (3) is applicable down to very small $x$. In terms of LHC parton kinematics, this corresponds to perturbative stability for central rapidities $|y| \lesssim 2$, while modifications are at most expected in the very forward (backward) regions $|y| \gtrsim 4$.

\subsection{W- and Z-boson production at LHC}

The immediate question arises: what is the impact of our current knowledge of parton distributions on the precision of LHC predictions, for instance for $W^{ \pm}, Z$-boson rapidity distributions, which often have been considered 'standard candle' processes for the parton 

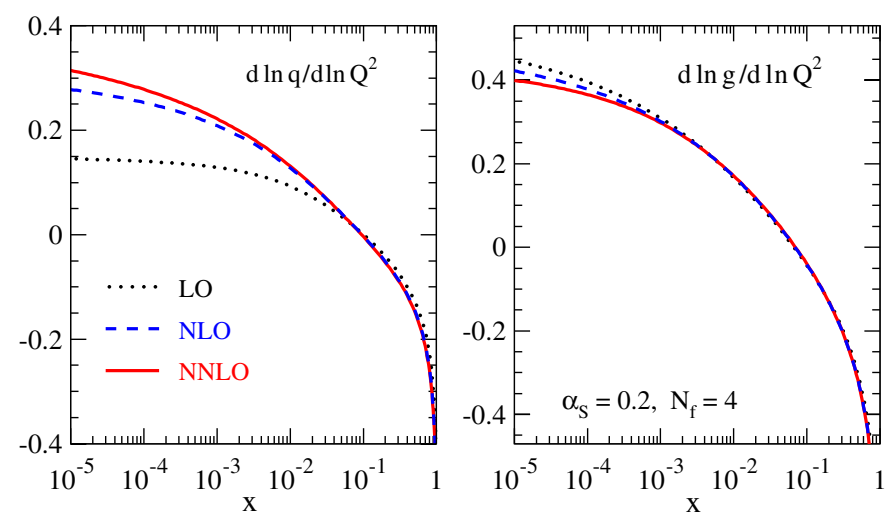

Figure 6. Perturbative expansion of the scale derivatives of typical quark and gluon distributions at $\mu^{2} \approx 30 \mathrm{GeV}^{2}$ (from [33], where the initial conditions are specified).
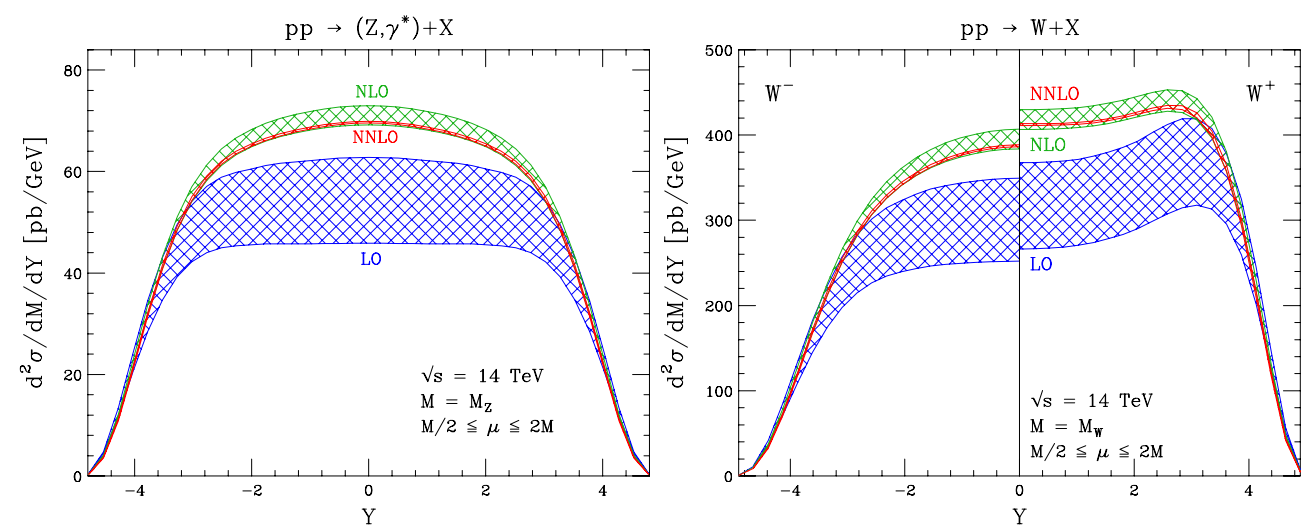

Figure 7. The rapidity-dependent cross sections for gauge-boson production at the LHC, using the partons of [46] and estimates of the theoretical uncertainty from variations of the scale $\mu$ (Reprinted with permission from 2004 Phys. Rev. D 69 094008. Copyright (2004) by the American Physical Society).

luminosity [38, 45]. The corresponding cross sections are known to NNLO in perturbative QCD, and according to equation (2) one can quantify the theoretical uncertainties obtained by varying the renormalization and factorization scale $\mu$ by the conventional (although arbitrary) factor of 2 around $M_{W, Z}$. The perturbative stability of the results in figure 7 nicely demonstrates the necessity of considering higher order perturbative corrections through NNLO in QCD. It would be impossible to make precision predictions, or perform precision analyses, based solely on the rough (and non-overlapping) LO and NLO error estimates.

Recent improvements in the parameterizations of PDFs, though, have been shown to significantly affect predictions for physical cross sections at LHC. An independent treatment of the strange quark distributions $f_{s}$ and $f_{\bar{s}}$ (hence their uncertainties), for instance, has an impact on the correlated uncertainties of the light sea quarks, because neutral current deepinelastic data on $F_{2}$ constrain the combination $4 / 9\left(f_{u}+f_{\bar{u}}\right)+1 / 9\left(f_{d}+f_{\bar{d}}+f_{s}+f_{\bar{s}}\right)$. In consequence, the size of the uncertainty on the sea quarks for values $x \sim 10^{-3}-10^{-2}$ at hard scales $Q^{2} \sim M_{W}^{2}$ roughly doubles from $\sim 1.5 \%$ to $\sim 3 \%$ for the MSTW group [50, 51]. CTEQ 

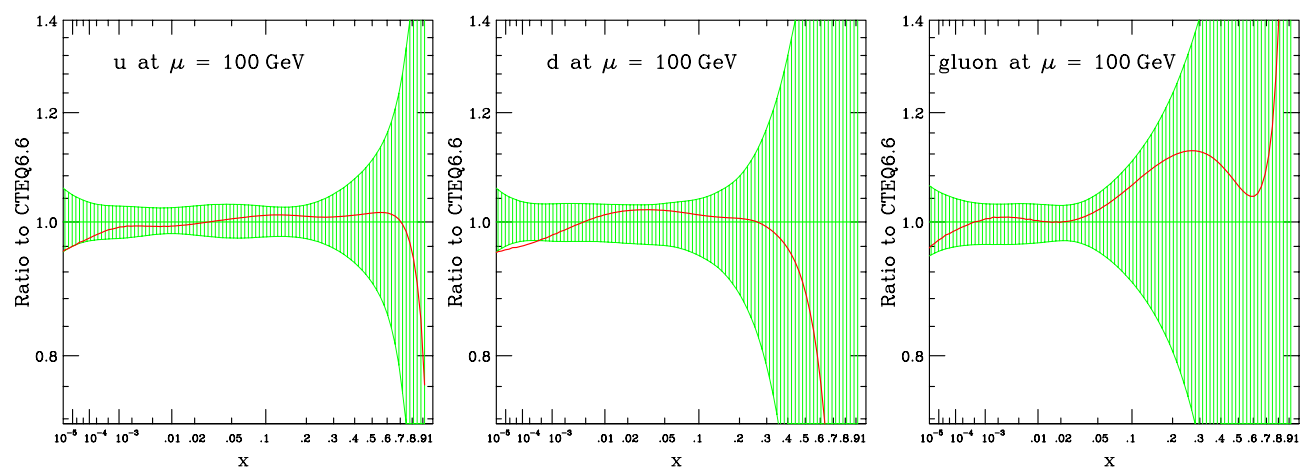

Figure 8. The distributions $f_{u}, f_{d}$ and $f_{g}$ of the CTEQ6.6 fit [48] with the estimated error bands (shaded area) at the scale $\mu=100 \mathrm{GeV}$. Comparison with previous results (CTEQ6.1M) [49] is denoted by the solid lines (Reprinted from Preprint arXiv:0802.0007 with permission from P M Nadolsky).

in their newer sets (e.g. CTEQ6.6) has improved the treatment of the charm contribution to the deep-inelastic structure function $F_{2}$ at HERA by implementing now a general-mass formalism for a variable flavor number scheme consistent with QCD factorization, see [52, 53]. The reduced charm component of $F_{2}$ is compensated by larger light quark distributions $f_{u}$ and $f_{d}$ at small $x$ as illustrated in figure 8 .

As an upshot, the predictions for $W^{ \pm}$- and $Z$-production cross sections at LHC being sensitive to PDFs in the $x \sim 10^{-3}$ range shift by $8 \%$ between the sets CTEQ6.6 [48] and CTEQ6.1M [49]. Although this particular shift originates from theoretical improvements long overdue, it is an example that PDFs and their associated uncertainties will have a significant impact on the precision of the 'gold-plated' $W^{ \pm}$and $Z$ cross sections and $W^{ \pm} / Z$ ratio calibration measurement. In this context, it should also be stressed that PDF uncertainties in the region of very small momentum fractions, $x \simeq 10^{-5}$ (as, e.g., displayed in figure 8), largely rely upon extrapolations of data and represent a certain parameterization bias.

\subsection{Parton distributions and the search for new physics}

Apart from gauge-boson production, there are prominent measurements at LHC which depend on our knowledge of parton distributions and, in turn, might be used to improve it. High- $E_{t}$ jet cross-sections, for instance, are a particularly prominent place to look for BSM effects. The discovery of new physics, such as e.g. jet signals for low mass strings [54], large extra dimensions or models parameterized in terms of contact interactions, becomes sensitive to the uncertainty of the gluon PDF especially at low- $x$. Recently, top-pair production has also been proposed as an additional calibration process at LHC, because its PDF dependence is anti-correlated with Z-boson production [48] and correlated with Higgs boson production, especially for larger Higgs masses. Presently, however, the sizable theoretical uncertainties at NLO in QCD are limiting the applicability of this proposal.

For di-jet rates at LHC, e.g. the consequences for predictions from large extra dimensions, have been analyzed [55] through a modified renormalization group equation for the strong coupling $\alpha_{s}$, where the running of $\alpha_{s}$ accelerates due to power corrections as the compactification scale $M_{c}$ of the large extra dimensions is approached. Results of a study for the di-jet transverse momentum distribution $\left(p_{t}\right)$ with the event generator Pythia are displayed in figure 9. The plot clearly indicates the reduced sensitivity to extra dimensions because of 


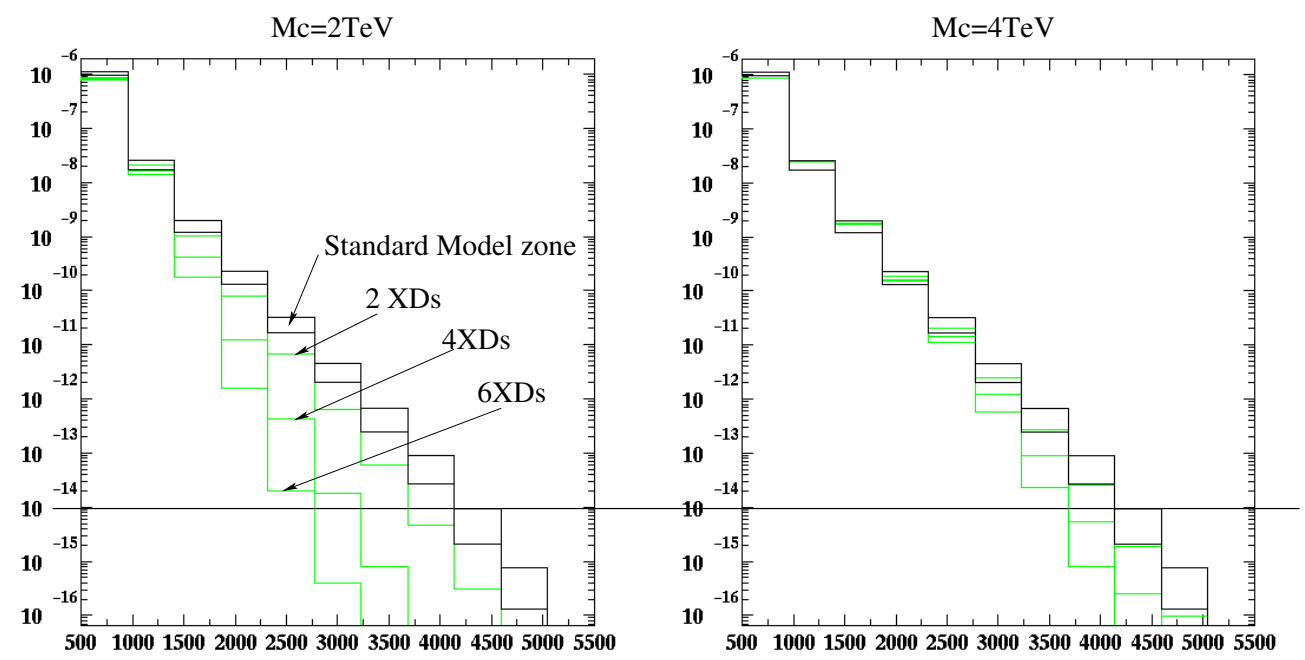

Figure 9. Di-jets cross sections at LHC as a function of $p_{t}$ for two compactification scales $M_{c}=$ $2 \mathrm{TeV}$ (left) and $M_{c}=4 \mathrm{TeV}$ (right) of the extra dimensions. Shown are different predictions for various numbers of extra dimensions, i.e. 2, 4 and 6, and the Standard Model zone incorporates the PDF uncertainties of the CTEQ6.1M set. The horizontal line shows the sensitivity limit corresponding to an LHC luminosity of $100 \mathrm{fb}^{-1}$ (Reprinted from Preprint hep-ph/0407303 with permission from S Ferrag).

the PDF uncertainties in the di-jet $p_{t}$-distribution. Hence there is need to either look at ratios of rates, $\sigma_{p p \rightarrow 3 \text { jets }}$ over $\sigma_{p p \rightarrow 2 \text { jets }}$, or else at angular correlations of di-jets to reduce the parton luminosity dependence.

On the other hand, PDF uncertainties most likely do not affect the discovery potential of a Higgs in the mass range $100-1000 \mathrm{GeV}$ or a high mass $Z^{\prime}$ in the mass range 150 $2500 \mathrm{GeV}$. Apart form the hadronic di-jets, promising other measurements to be conducted at LHC itself also include direct photon production to constrain the gluon PDF at low- $x$ or the $W^{ \pm}$-asymmetry to obtain information on the low- $x$ valence PDFs. In particular, the improved description of the (anti-)strange quark distributions leads to interesting implications for collider phenomenology. For instance the production of a charged Higgs boson $\mathrm{H}^{+}$via the partonic process $c+\bar{s} \rightarrow H^{+}$provides an example of a BSM process that is sensitive to the strange PDF in models with two or more Higgs doublets. The cross section also depends on a possible intrinsic charm component of the proton and the recent PDF set CTEQ6.5c provides various models for such a component [56].

\section{Parton cross sections}

Let us next turn to the hard-scattering cross sections initiated through the constituent partons $i, j$ of the incoming protons. In general, we consider $\hat{\sigma}_{i j \rightarrow X}$ where $X$ denotes any final state allowed by the Standard Model or its possible extensions. Calculations of $\hat{\sigma}_{i j \rightarrow X}$ result in predictions for experimental signatures and, eventually, determine the power to discriminate BSM signals or, e.g. Higgs boson production, from known background of the Standard Model. The key issue for QCD theory is the reliability of signal and background estimates. 
Table 2. Scattering processes at LHC for which the radiative corrections to NLO in QCD are needed, as summarized in Les Houches 2005 (from [62]).

\begin{tabular}{lll}
\hline Process $\left(V \in\left\{\gamma, W^{ \pm}, Z\right\}\right)$ & Background to & Reference \\
\hline$p p \rightarrow V V+1$ jet & $t \bar{t} H$, new physics & $W W+1$ jet [57, 58] \\
$p p \rightarrow H+2$ jets & $H$ production by vector-boson fusion (VBF) & $H+2$ jets [59] \\
$p p \rightarrow t \bar{t} b \bar{b}$ & $t \bar{t} H$ & \\
$p p \rightarrow t \bar{t}+2$ jets & $t \bar{t} H$ & \\
$p p \rightarrow V V b \bar{b}$ & $\mathrm{VBF} \rightarrow V V, t \bar{t} H$, new physics & \\
$p p \rightarrow V V+2$ jets & $\mathrm{VBF} \rightarrow V V$ & \\
$p p \rightarrow V+3$ jets & Various new physics signatures & ZZZ [60], WWZ [61] \\
$p p \rightarrow V V V$ & SUSY trilepton &
\end{tabular}

\section{1. $Q C D @ N L O$}

At a hadron collider, the problem of signal significance has various aspects. Experimentally, measurements of hard-scattering reactions require reliable identification of leptons (electrons, muons), a good understanding hard jets at high transverse momentum $\left(p_{t}\right)$, especially $b$-quark jets, and a sufficiently precise calibration of the jet energy scale. Moreover, BSM or Higgs searches heavily rely on the presence of large missing transverse energy $\left(E_{t}\right)$ to reject Standard Model background compared to the signal.

On the theory side, as briefly mentioned in section 2 , we have various levels of accuracy for the hard-scattering process (assuming that the underlying event and multiple parton interaction are modeled by shower Monte Carlos). Estimates to LO in QCD based on exact matrix elements seem mandatory in search scenarios for studies of distributions, e.g. in $p_{t}$ or the (pseudo-)rapidity $(\eta)$ and for assessing the effects of kinematical cuts. It is well known that the overall normalization and, in particular, the hard tail of these distributions (e.g. at high $p_{t}$ or $E_{t}$ ) are not well modeled by shower Monte Carlos alone. However, any LO prediction has large theoretical uncertainties, typically estimated by the scale variation, equation (2). Consider, for instance, the cross section for $p p \rightarrow W+4$ jets, which is of $\mathcal{O}\left(\alpha_{s}^{4}\right)$ at LO. From a variation of the coupling of $\Delta\left(\alpha_{s}^{\mathrm{LO}}\right) \simeq 10 \%$ one can roughly estimate a cross-section uncertainty of $\Delta\left(\sigma^{\mathrm{LO}}\right) \simeq 40 \%$. Thus, one needs to go beyond the Born approximation for certain processes.

Perturbative QCD corrections at NLO to scattering processes are essential for the rates and shapes of distributions of Standard Model processes as well as for BSM searches, where they may have an impact on the signal significance. Often, one encounters large $K$-factors and also new parton channels open up at NLO which may eventually dominate beyond tree level, a prominent example being single-top production (see section 5.2). In a series of workshops a number of key processes at LHC have been identified which need to be known to NLO in QCD. These are summarized in the so-called LHC 'priority' wishlist in table 2 and the computation of these radiative corrections is presently a very active field of research.

The obvious question is, of course, why the calculation of one-loop corrections in QCD is so difficult? After all, the conceptual issues are all solved and any computation can follow a straightforward algorithm: draw all Feynman diagrams and evaluate them, then use standard reduction techniques for tree and loop amplitudes. While this is true in principle, it is hard in practice with known bottlenecks, because intermediate expressions are much more complicated than the final result. Thus, let us look at the outline of a generic NLO calculation as displayed in figure 10. For the scattering reaction $2 \rightarrow n$ partons, the basic ingredients in the cross-section calculation are the real corrections, i.e. the tree level $2 \rightarrow(n+1)$ parton 


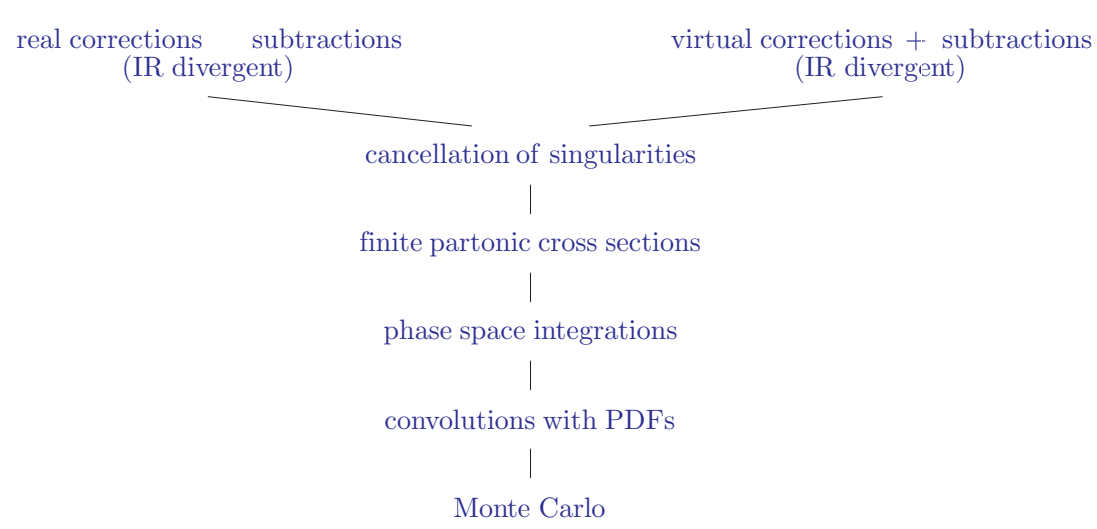

Figure 10. Outline of a generic calculation of NLO QCD corrections to a (multi-particle) scattering process in the traditional approach.

reaction $\mathrm{d} \sigma^{\text {real }}$, and the one-loop virtual corrections to the $2 \rightarrow n$ parton amplitude, which are subject to the standard ultraviolet (UV) renormalization.

The latter contribution to the cross section, that is the one-loop virtual correction $\mathrm{d} \sigma^{\text {virtual }}$, in the standard Feynman diagram approach generates large expressions, although we do expect large cancellations between the diagrams in a gauge theory as a consequence of gauge invariance. Specifically, one is required to calculate tensor integrals like, e.g.

$$
I^{\mu_{1}, \mu_{2}, \ldots}\left(k_{1}, \ldots\right)=\int \mathrm{d}^{D} p_{1} \frac{p_{1}^{\mu_{1}} p_{2}^{\mu_{2}} \cdots}{\left(p_{1}^{2}-m_{1}^{2}\right)\left(\left(p_{1}-k_{1}\right)^{2}-m_{2}^{2}\right) \cdots} .
$$

Unfortunately, these become rather complicated for five or more external particles, one wellknown problem being the numerical stability for all allowed configurations of the external momenta. Without going into details here, suffice it to say that reduction algorithms for tensor integrals are some 30 years after the work of Passarino and Veltman [63] still a very active field of research, see e.g. [64-66]. Moreover, no completely general libraries are available here (see, e.g., LoopsTools [67] for public code).

Coming back to figure 10 , a characteristic feature of both contributions $\mathrm{d} \sigma^{\text {real }}$ and $\mathrm{d} \sigma^{\text {virtual }}$ is the presence of infrared (IR) divergencies due to soft and collinear regions in phase space. The physical cross section $\sigma_{2 \rightarrow n}^{\mathrm{NLO}}$ being the sum of both is, of course, IR finite after absorbing the initial-state collinear singularities into the PDFs by mass factorization (see, e.g., [68] for details). As we aim at parton level Monte Carlos to NLO accuracy with a flexible phase-space integration allowing for kinematical cuts, the IR divergencies need to be treated accordingly. Among the many proposed methods (see, e.g., [8]), the so-called dipole subtraction [69, 70] has emerged as a standard procedure. Here, the cancellation of infrared singularities due to collinear partons or soft gluons is implemented 'locally' by subtracting (over the entire phase space) functions (the dipoles) that approximate the singularities of the real emission part. Subsequently, the integrated dipoles are added to the virtual corrections. Employing dimensional regularization $(d=4-2 \epsilon)$, the master formula reads $[69,70]$

$\sigma_{2 \rightarrow n}^{\mathrm{NLO}}=\int_{n+1}\left[\left(\mathrm{~d} \sigma^{\text {real }}\right)_{\epsilon=0}-\left(\mathrm{d} \sigma^{\text {dipole }}\right)_{\epsilon=0}\right]+\int_{n}\left[\mathrm{~d} \sigma^{\text {virtual }}+\int_{1} \mathrm{~d} \sigma^{\text {dipole }}\right]_{\epsilon=0}$,

which is understood to also contain the mass factorization of the remaining initial-state collinear singularities. With equation (6) one arrives at a finite partonic cross section $\sigma_{2 \rightarrow n}^{\mathrm{NLO}}$ 
which can be integrated numerically over the available phase space and convoluted with the PDFs. Let us stress, however, that for any practical solution of equation (6) speed and stability of the numerics are criteria of paramount importance. Quite often, for instance, the programs for a particular scattering process need dedicated optimization. Thus, presently there is a lot of room for technological progress with respect to automatization and algorithms in order to fill the empty spaces in table 2.

\subsection{New theory developments}

One specific direction of research in theory during the past few years has been toward new analytic techniques to calculate gauge theory amplitudes. The specific focus has been on a recursive approach in which all intermediate quantities are on-shell and hence gauge invariant (see [71] for a recent review). As a matter of fact, techniques for computing tree amplitudes recursively are well established since a number of years [72]. Moreover, it has been realized that an efficient management of the quantum numbers for a given scattering amplitude reduces the computational complexity by far. The known methods include the so-called color ordering, the use of helicity amplitudes and a decomposition of QCD amplitudes exploiting effective supersymmetry (SUSY) (see, e.g., [73]). In addition, factorization properties of amplitudes in the soft and collinear limits serve as a strong check (see for instance $[69,73]$ ).

In a helicity basis, amplitudes for scattering processes are classified according to the number of ' \pm ' -states of the external partons. The so-called maximal helicity violating (MHV) amplitude denotes the configuration with the largest difference of ' + '- and '-'-states, e.g. $n-2$ for an $n$-gluon amplitude $A_{n}$ at tree level. The tree level (color ordered) $n$-gluon MHV amplitude $A_{n}^{\text {tree }}$ takes a particularly simple and elegant form [74]. In terms of (Weyl) spinor inner products $\langle j l\rangle=\overline{u_{-}}\left(k_{j}\right) u_{+}\left(k_{l}\right)$ for massless Weyl spinors $u_{ \pm}(k)$ of momentum $k$ we can write (see also, e.g., [75]),

$$
A_{n}^{\text {tree }}\left(1^{-}, 2^{-}, 3^{+}, \ldots, n^{+}\right)=\mathrm{i} \frac{\langle 12\rangle^{4}}{\langle 12\rangle\langle 23\rangle \ldots\langle n 1\rangle},
$$

which is in a certain sense 'all-order' information, because equation (7) holds for any number $n$ of external gluons.

The importance of helicity amplitudes became again apparent upon applying twistor space methods [76] and by realizing that tree amplitudes $A_{n}^{\text {tree }}$ in gauge theories possess unique analytic properties which become manifest, if considered as functions of complex momenta $k$, i.e. under a shift $k \rightarrow k(z)$ for a complex-valued parameter $z$. These analyticity properties of $A_{n}^{\text {tree }}(z)$ can be turned into recursion relations for the case of gluons [77, 78] as well as quarks and scalars [71, 79, 80], which allow the construction of $n$-point helicity amplitudes from on-shell $(n-2)$-point amplitudes. From $A_{n}^{\text {tree }}(z)$ the physical amplitude at $z=0$, i.e. $A_{n}^{\text {tree }}(0)$, can be reconstructed simply by exploiting Cauchy's theorem of complex analysis. The key feature of complex kinematics is the fact that the three-parton primitive MHV amplitude, e.g. equation (7) for $n=3$, does not vanish for on-shell complex momenta $k(z)$, while it does so for real $k$. For details of the complex shift $k \rightarrow k(z)$ we refer to [78].

The real quest, of course, has been in devising improved methods for the calculation of one-loop corrections to scattering amplitudes. This has been a very active field of research over the past few years and it has been realized that unitarity methods provide the additional key ingredient here. Unitarity is a fundamental concept in quantum field theory which manifests itself for instance in the Cutkosky cutting rules for Feynman diagrams (related to the optical theorem). In the computation of one-loop amplitudes, unitarity appears as fusing rules for amplitudes [71, 82]. One aims at reconstructing the real part of a given one-loop amplitude 


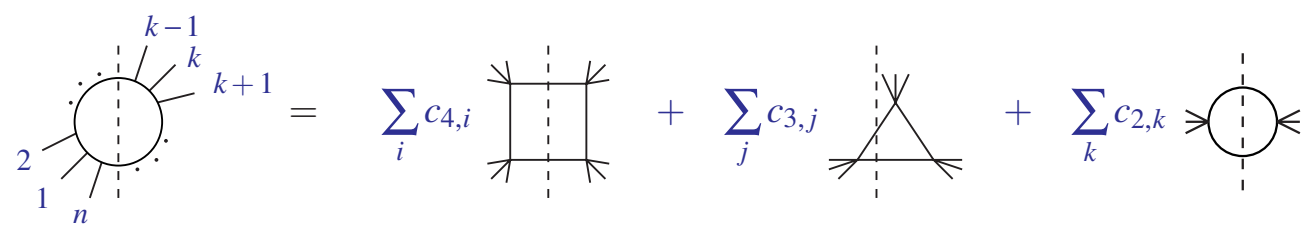

Figure 11. Schematic representation of the unitarity approach: sewing of tree level amplitudes leads to the imaginary part of one-loop $n$-point amplitudes. The real part is determined subsequently from the analytic properties of the amplitudes (adapted from [81]).

from the imaginary one by sewing together tree level amplitudes. To that end, one uses the fact that any one-loop amplitude can be expressed in a basis of scalar integral functions, i.e. boxes, triangles and bubbles. This is obvious from the standard reduction techniques, e.g. [63], and it is sketched schematically in figure 11. Unitarity cuts then allow to uniquely identify the contribution to the individual integral (i.e. the coefficients $c_{4, i}, c_{3, j}, c_{2, k}$ in figure 11) from its imaginary part, for example a triangle loop from calculating $i \pi \ln (-s) \rightarrow \ln ^{2}(-s)$.

Generalized unitarity (see, e.g., [83]) developed further the idea of reconstructing the coefficients $c_{4, i}$, etc by imposing quadruple cuts, which constrain all components of a given one-loop integral (5). As an upshot, all terms with logarithmic dependence are cutconstructible. One-loop QCD amplitudes, however, also contain rational (non-logarithmic) terms, which are rather difficult to derive and require substantially more effort.

To that end, as a further principle to organize the calculation a SUSY-inspired decomposition of one-loop amplitudes has been very useful. For the amplitude $A_{n}^{g}$ with $n$ external gluons and a gluon circulating in the loop we can write

$$
A_{n}^{g}=\underbrace{\left(A_{n}^{g}+4 A_{n}^{f}+3 A_{n}^{s}\right)}_{\mathcal{N}=4 \text { SUSY }}-4 \underbrace{\left(A_{n}^{f}+A_{n}^{s}\right)}_{\mathcal{N}=1 \text { chiral SUSY }}+\underbrace{A_{n}^{s}}_{\mathcal{N}=0 \text { scalar }},
$$

where the internal loop degrees of freedom are ordered in terms of supersymmetric multiplets, i.e. the $\mathcal{N}=4$ multiplet (one gluon, four Weyl fermions, six real scalars), the chiral $\mathcal{N}=1$ multiplet (one Weyl fermion, two real scalars) and the $\mathcal{N}=0$ part with a complex scalar in the loop. This has the great advantage that $\mathcal{N}=4$ and the $\mathcal{N}=1$ contributions are completely cut-constructible. For the rational part in the $\mathcal{N}=0$ bit, more involved recursions have been developed.

Finally, the computation of the one-loop virtual corrections to the six gluon amplitude has been completed through the effort of many groups [84-95] (see table 3) and the analytic results have been confirmed for specific phase-space points by a completely numerical evaluation [96]. Recently, the numerical approach has been developed further and shown to have promising potential [97]. We therefore expect that the NLO correction to the four-jet cross section at LHC is within sight.

Let us end the discussion by mentioning a few directions for further development. Clearly, the new techniques have to be employed in complete cross-section calculations following the steps outlined in section 4.1. Moreover, at LHC many processes of interest contain either gauge boson or bottom and top quarks (see table 2). Thus, the formalism sketched above needs to be carried over to the case of massive (colored) particles. This requires a number of extensions, be it the helicity formalism or the methods for calculating massive one-loop integrals from generalized unitarity cuts (see, e.g., [98, 99]). We should also mention that of course many other developments in theory have pushed the precision frontier for QCD 
Table 3. The analytic analytic computation of the one-loop QCD corrections to the individual helicity configurations of the six-gluon amplitude in the decomposition of equation (8) as a community effort (adapted from [81]).

\begin{tabular}{lllll}
\hline \multirow{2}{*}{ Amplitude } & $\mathcal{N}=4$ & $\mathcal{N}=1$ & $\begin{array}{l}\mathcal{N}=0 \\
\text { cut }\end{array}$ & $\begin{array}{l}\mathcal{N}=0 \\
\text { rat }\end{array}$ \\
\hline--++++ & {$[84]$} & {$[85]$} & {$[85]$} & {$[90]$} \\
-+-+++ & {$[84]$} & {$[85]$} & {$[87]$} & {$[94,95]$} \\
-++-++ & {$[84]$} & {$[85]$} & {$[87]$} & {$[94,95]$} \\
---+++ & {$[85]$} & {$[86]$} & {$[91,92]$} & {$[93]$} \\
--+-++ & {$[85]$} & {$[88,89]$} & {$[92]$} & {$[95]$} \\
-+-+-+ & {$[85]$} & {$[88,89]$} & {$[92]$} & {$[95]$} \\
\hline
\end{tabular}

predictions further, be it for multi-loop calculations (NNLO and beyond), for resummations or simply for algorithms and tools. Unfortunately we could not touch those aspects.

\subsection{Complete $N L O$ and $N N L O$ results}

Let us conclude this section by summarizing the state-of-the-art for complete NLO crosssection predictions for many-particle production. It has become clear in the preceding discussion (see table 2) that current theory research is focused on processes with $2 \rightarrow 3$ and $2 \rightarrow 4$ potentially massive particles like top quarks, $W / Z$-bosons, etc.

A few outstanding results that have appeared in the last one or two years in this respect address for instance Higgs production in the Standard Model. Here a number of reactions have been investigated at NLO, such as $p p \rightarrow H+2$ jets via gluon fusion [59] (in the heavy top limit, see section 6) and via weak interactions [100] as well as $p p \rightarrow H+3$ jets in vectorboson fusion [101]. These results provide important information, e.g., for the extraction of the Higgs coupling to vector bosons at LHC. The production of vector bosons has been considered to NLO accuracy in QCD, e.g. for the reaction $p p \rightarrow V V+2$ jets via vector-boson fusion [102-104], for tri-boson production $(p p \rightarrow Z Z Z, W W Z$ ) [60, 61] and for $p p \rightarrow W W+$ 1 jet $[57,58]$. Especially, the latter case is an important background for Higgs production in the low mass range $(H \rightarrow W W)$ and subsequent semi-leptonic decay of the $W$-bosons. A largely complete list of NLO calculations for LHC processes including electroweak corrections and also a number of new physics signals with, e.g., the SUSY QCD corrections has been given in [62].

Some predictions for $p p \rightarrow 3$ particles are published as public codes. Many of these programs also provide continuous updates which are made available to the community, e.g., through the CEDAR project [105]. To mention a few explicitly, let us point out NLOJET++ $[20,21]$ which comes as a multipurpose C++ library for calculating jet cross sections at NLO, e.g. for three-jet rates in hadron collisions. The program MCFM [22] calculates for instance the production of a gauge boson or a Higgs in association with jets at hadron colliders. The PHOX family with DIPHOX [106] and JETPHOX [107] deals specifically with hard QCD radiation of photons along with jets in hadron collisions, which is another important background for the low mass Higgs, e.g. in the di-photon mode $(H \rightarrow \gamma \gamma)$. Predictions for the photon pair-production background including a resummation of the transverse momentum $p_{t}$ of the di-photon pair $[108,109]$ have been subject of the most recent improvements in the program ResBos [110].

NLO radiative corrections to scattering reactions with six external particles, i.e. $2 \rightarrow 4$ processes, constitute the current technological frontier. Benchmark results are the calculation 


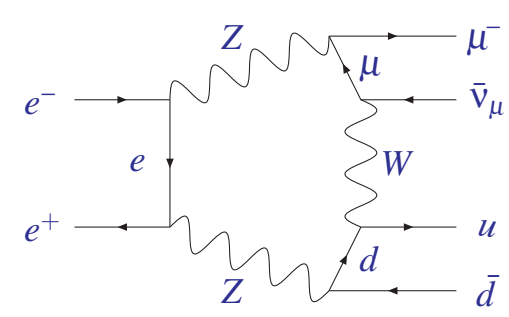

Figure 12. Sample of Feynman diagram (hexagon with internal masses) for electroweak corrections to $e^{+} e^{-} \rightarrow 4$ fermions.

of the complete electroweak corrections to $e^{+} e^{-} \rightarrow 4$ fermions [111, 112]. This reaction involves at the loop level extremely difficult hexagon integrals with masses, see figure 12 . Of similar complexity are the NLO electroweak corrections to Higgs production in association with a neutrino pair, $e^{+} e^{-} \rightarrow v \bar{v} H H$, obtained by the GRACE group [113,114]. Also the NLO QCD corrections to the combined production of a top and a bottom pair, i.e. the process $\gamma \gamma \rightarrow t \bar{t} b \bar{b}$, are known [115].

Finally, there is of course demand for fully differential QCD predictions to NNLO for hadron collider processes. As mentioned above, this scope has been achieved, e.g., for the dilepton pair production in Drell-Yan [28] or Higgs production in gluon fusion [29] together with the parton evolution $[32,33]$. However, it remains a challenge for hadronic di-jet production, where large statistics even with high- $p_{t}$ cuts is anticipated at LHC. The measurement of gluon jets would constrain for instance the gluon PDF at medium- and large- $x$ and di-jet angular correlations are important observables in BSM searches for quark sub-structure. NNLO predictions for di-jets are likely to reduce the scale uncertainty and to improve the modeling of jets. Recent extensions of table 2 also list the NNLO corrections to Higgs production in vector-boson fusion, to top-pair production and to $V+1$ jet, where $V \in\left\{\gamma, W^{ \pm}, Z\right\}$.

Unfortunately, the calculation of NNLO cross sections is very difficult. Although many (two-loop) virtual amplitudes are known since some years, the cancellation of IR divergencies between virtual and real corrections remains highly non-trivial and the numerical phase-space integration very difficult, i.e. the NNLO equivalent of steps outlined in figure 10. This is a vast subject on its own, which we will not pursue further here (see, e.g., [8] for a brief review). Suffice it to say that progress in this direction has been achieved only recently for the differential distributions in the case of $e^{+} e^{-} \rightarrow 3$ jets [116, 117].

\section{Top quark production at LHC}

Top quarks will be copiously produced at LHC. For the pair-production mode the collider will accumulate very high statistics of approximately $8 \times 10^{6}$ events with $t \bar{t}$-pairs with $10 \mathrm{fb}^{-1}$ per year in the initial low luminosity run $[2,3]$. These data will allow for numerous measurements, e.g. of the top-mass, where the experiments aim at an accuracy of $\Delta m_{t}=\mathcal{O}(1) \mathrm{GeV}$, and also for tests of the production and the subsequent decay mechanism including anomalous couplings and top-spin correlations (see, e.g., [118]). Top quark decay $(t \rightarrow W b$ ) leads to very characteristic signatures allowing for event reconstruction in many channels through the observed leptons ( $b$-flavored), jets and missing $E_{t}$. In general, top quarks make up large part of background for Higgs production or BSM searches. Moreover, due to the large mass (currently $m_{t}=170.9 \pm 1.1$ (stat) +1.5 (syst) GeV, see [119]) close to the scale of electroweak 


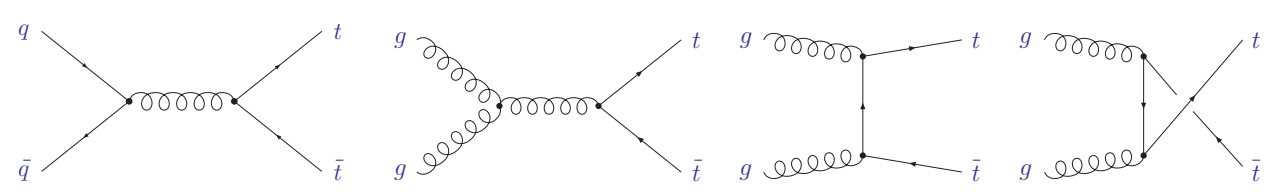

Figure 13. Complete set of Feynman diagrams to leading order for the heavy-quark pair production in light quark annihilation, $q+\bar{q} \rightarrow t+\bar{t}$ (left), and in gluon fusion $g+g \rightarrow t+\bar{t}$ (three diagrams on the right).
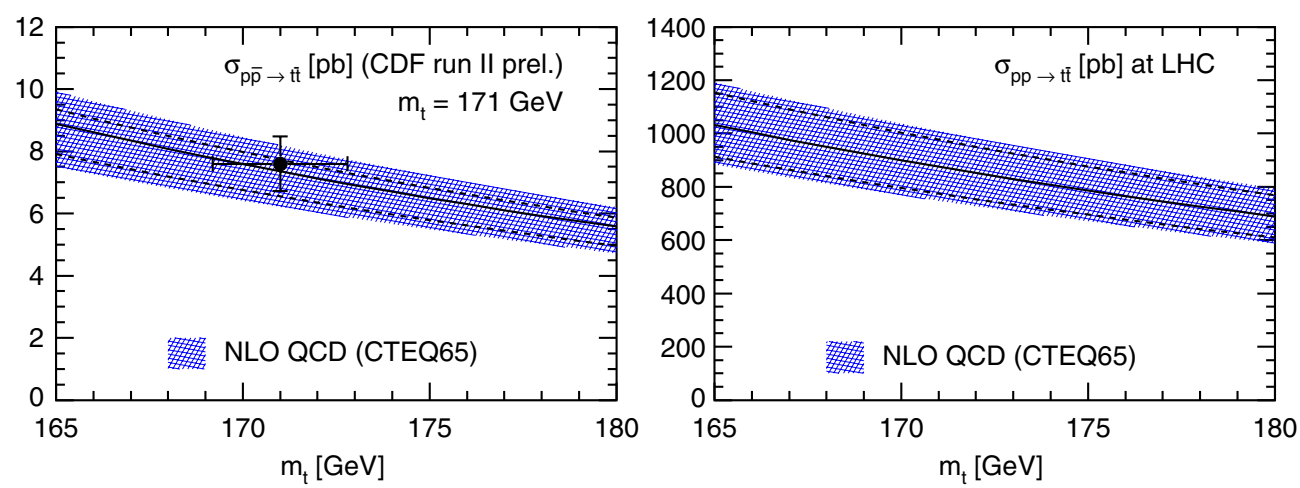

Figure 14. The $t \bar{t}$ total cross section to NLO QCD as a function of $m_{t}$ for Tevatron at $\sqrt{S}=$ $1.96 \mathrm{TeV}$ and CDF data [123] for $m_{t}=171 \mathrm{GeV}$ (left) and LHC at $\sqrt{S}=14 \mathrm{TeV}$ (right). The solid line is the central value for $\mu=m_{t}$, the dashed lower and upper lines correspond to $\mu=2 m_{t}$ and $\mu=m_{t} / 2$, respectively. The band denotes the additional PDF uncertainty of the CTEQ6.5 set [124].

symmetry breaking top quarks play a prominent role in many new physics scenarios (see, e.g., [120]).

\subsection{Top quark pair production}

The hadronic heavy-quark pair production is known to NLO in QCD for many years $[121,122]$ and it still serves as an example to illustrate many generic features of QCD corrections to the production of heavy colored particles, see figure 13 for the corresponding Feynman diagrams to leading order. Depending on the collider, i.e. Tevatron $(p \bar{p})$ or LHC $(p p)$, the parton luminosities enhance the respective parton channels $q \bar{q}$ and $g g$. Thus, at Tevatron, $q \bar{q}$-annihilation saturates the total cross section to $\mathcal{O}(90 \%)$, while at LHC a similar dominance of gluon fusion holds. The channels $q g(\bar{q} g)$ newly opening up at NLO contribute only $\mathcal{O}(1 \%)$ at the scale $\mu=m_{t}$ at both colliders.

In figure 14 , we show the NLO QCD predictions for the total cross section of $t \bar{t}$-pair production at Tevatron and LHC. The band denotes the scale variation in the usual range $\left(m_{t} / 2 \geqslant \mu \geqslant 2 m_{t}\right)$ as well as uncertainties related to the parton luminosity. At Tevatron, the theory error budget at NLO in QCD is slightly asymmetric $+12 \% /-15 \%$ which breaks down to a scale uncertainty of $+5 \% /-10 \%$ and a PDF uncertainty of $+7 \% /-5 \%$. The latter one is due to the PDFs being sampled in the large- $x$ region, where especially the gluon is poorly constrained. At LHC, the total theory error is $15 \%$ which consists of a scale uncertainty of $11 \%$ and a much smaller PDF uncertainty of $4 \%$. Here, the cross section is sensitive to the 

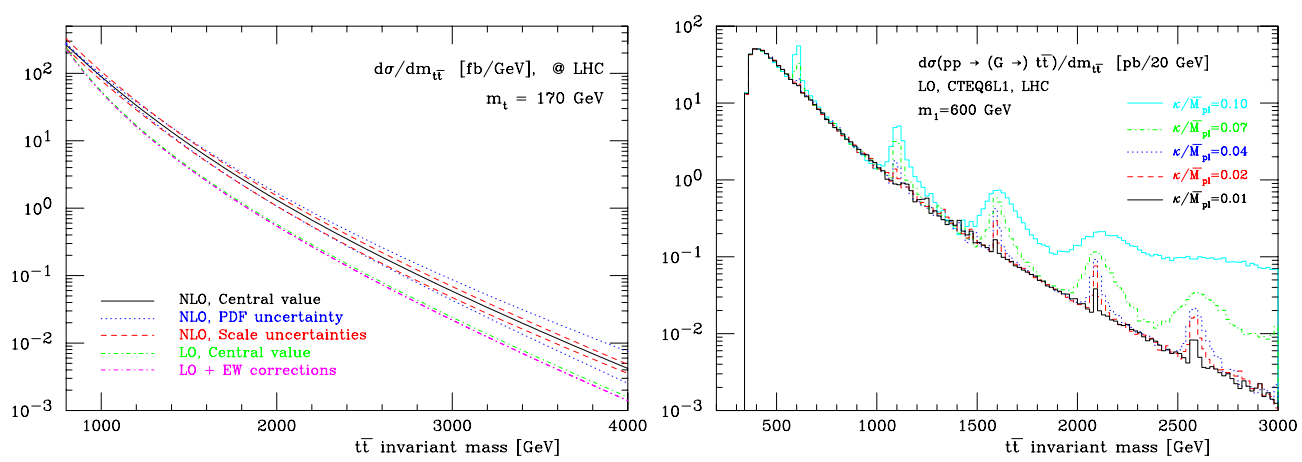

Figure 15. Left: the $t \bar{t}$ invariant mass spectrum at LHC for $m_{t}=170 \mathrm{GeV}$ at NLO in QCD together with the scale (dashed) and the PDF (dotted) uncertainties for the CTEQ6.1M set. Also plotted are predictions at LO in QCD (normalized to the NLO total cross section) with (dark dash-dotted) and without (light dash-dotted) the NLO electroweak corrections for the CTEQ6L1 set (Reprinted from Preprint arXiv:0712.2355 with permission from R Frederix and F Maltoni). Right: the $t \bar{t}$ invariant mass spectrum at LHC including $s$-channel graviton exchange and the effect of a couple of Kaluza-Klein resonances in an extra dimensions model (Reprinted from Preprint arXiv:0712.2355 with permission from R Frederix and F Maltoni).

gluon PDF in a range well covered by HERA (see figure 5). Different sets of global PDFs agree within the given error bands, although it should be pointed out that there can be sizable shifts in the central values. For example, there is a $3 \%$ shift in the central value between the CTEQ6.5 and CTEQ6.6 sets [48] with correct heavy flavor treatment and the older set CTEQ6.1M (see also [125] for a recent discussion).

The theoretical prediction can be improved in specific kinematical regions. Near threshold a Sudakov resummation can be performed [126-128], which stabilizes perturbative predictions if the $t \bar{t}$-pairs are produced close to partonic threshold as for instance at Tevatron and, perhaps, to a lesser extent at LHC. Further improvements of the theoretical accuracy need the NNLO QCD corrections, which are mandatory for a precision of better than $\mathcal{O}(10 \%)$ as envisaged by the LHC experiments. First steps in this direction have been undertaken by evaluating the interference of the one-loop QCD corrections [129] and by deriving the virtual contributions to heavy-quark hadro-production at two loops in the ultra-relativistic limit $m^{2} \ll s, t, u$ $[130,131]$ based on a simple relation of massive and massless amplitudes in the limit $m \rightarrow 0$ [132] (see also the review [133]). A precise understanding of the kinematical region $m \rightarrow 0$ beyond NLO is of immediate relevance also bottom-pair production over a large kinematical range and heavy flavor production at large $p_{t}$.

Top quark mass determinations at LHC are usually planned to proceed through direct reconstruction because the theoretical accuracy of the total cross section is presently insufficient, see figure 14. An interesting alternative (see, e.g., [3, 4]) involves a mass measurement through $J / \psi$ final states from $b$-decays in the top quark decay chain $(t \rightarrow W b)$. The $J / \psi$-reconstruction is supposed to give an accurate measurement of the $b$-quark momentum thanks to the relatively high mass of the meson. However, currently, it is also limited by our knowledge of the heavy-quark fragmentation the perturbative description of which has been extended to NNLO in QCD only recently [134-137].

Other observables of experimental interest at LHC are differential distributions in the transverse momentum $p_{t}$ of an identified top quark or in the invariant mass $m_{t \bar{t}}$ of the $t \bar{t}$-pair (see, e.g., [120]). Predictions for the latter are displayed in figure 15 (left) based on NLO in QCD as calculated, e.g., with MCFM [22] and also including NLO electroweak corrections 

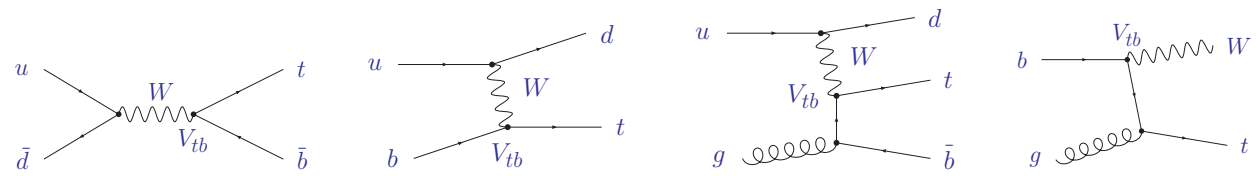

Figure 16. Sample of Feynman diagrams for single-top production. Shown are the production in the $s$-channel (left), $b$-initiated $t$-channel (second from left), $g$-initiated $t$-channel (second from right) and $b g$-channel (right).

[138] for the CTEQ6.1 set [49]. In the TeV-region for the invariant mass $m_{t \bar{t}}$ both the NLO QCD corrections and also the electroweak radiative effects grow. The dominant theoretical errors however come from the scale and the PDF uncertainties. In particular, the latter start to increase because the dominant contributions come again from the poorly known large- $x$ region.

It has been pointed out [120] that the $m_{t \bar{t}}$-distribution also provides a window to new physics, where $s$-channel resonances may become visible. Figure 15 (right) nicely illustrates the effect of graviton exchange in a model with one extra dimension compactified to a $\mathbf{S}^{1} / \mathbf{Z}_{2}$ orbifold (see, e.g., [139] and references therein). Such a model leads to a tower of KaluzaKlein modes giving rise to a series of resonances in the $t \bar{t}$ invariant mass spectrum, the details (peak position and width) of course, depending on the compactification scale and the effective coupling $\kappa / \bar{M}_{p l}$.

\subsection{Single-top quark production}

The interest in single-top production at hadron colliders comes from a number of reasons. It allows for studies of charged-current weak interactions of the top quark and for a direct extraction of the CKM-matrix element $V_{t b}$. Moreover, depending on the model under consideration, the cross section for single-top production acquires large corrections in BSM scenarios. At Tevatron first evidence for single-top production has been found only rather recently [140, 141].

The distinct channels for single-top production are displayed in figure 16, where samples of leading order Feynman diagrams are shown. The $s$-channel mode (figure 16, left) proceeds through the production of an off-shell $W$-boson and subsequent decay $(W \rightarrow t b)$. The initial state is proportional to the light flavor PDFs and the rate at LHC is relatively small. The $t$-channel exchange of a virtual $W$-boson in the boson-gluon fusion mode (figure 16, second from right) on the other hand is the dominant production mechanism, both at Tevatron and LHC. In the latter case, it is much enhanced due to the gluon PDF and subsequent splitting to a $b \bar{b}$-pair $(g \rightarrow b \bar{b})$, while the $b$-initiated $t$-channel process $u b \rightarrow d t$ itself (figure 16 , second from left) is suppressed by the numerically small bottom PDF. Of course, precise predictions at higher orders require a consistent matching of both processes, i.e. $g \rightarrow b \bar{b}$-splitting in the hard parton scattering and in the evolution [142]. For this main single-top production mode the NLO QCD corrections have also been subject of the latest addition to MC@ NLO [143], so that NLO parton level calculations and showering are consistently combined. Finally, there is $W t$-production in the $b g$-channel (figure 16, right) being the second largest mode at LHC, but with negligible rates at Tevatron due to limited phase space. The $b g$-channel is subleading in QCD (counting powers of the coupling constants) but again enhanced by the gluon luminosity.

Sensitivity to BSM models appears in different manifestations for the various channels of single-top production. If one allows for anomalous couplings or flavor changing neutral 

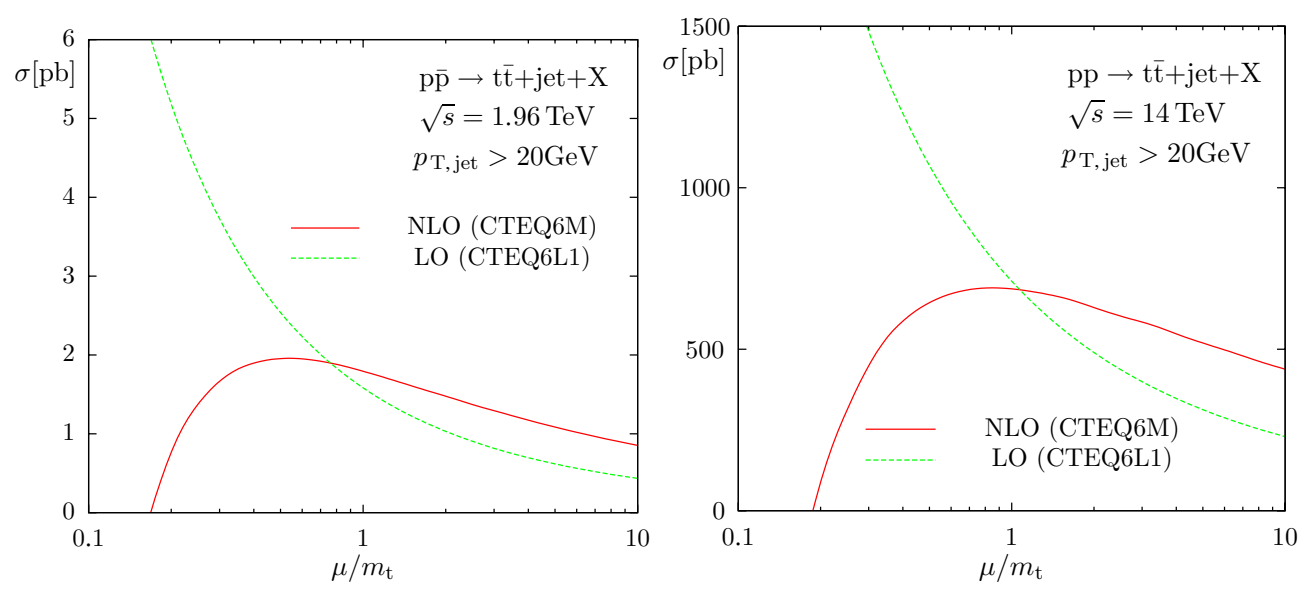

Figure 17. The scale dependence of the LO and NLO cross sections for $t \bar{t}+1$ jet production at the Tevatron (left) and at LHC (right) with renormalization and factorization scales identified, $\mu_{r}=\mu_{f}=\mu$ (Reprinted with permission from 2007 Phys. Rev. Lett. 98 262002. Copyright (2007) by the American Physical Society).

currents, the $t$-channel contribution is altered significantly. On the other hand, the existence of charged 'top-pions', excited Kaluza-Klein modes of the $W$-boson or, similarly, a $W^{\prime}$-boson would have impact on the $s$-channel.

\subsection{Top quark plus jet production}

The final example of this section is concerned with top quark plus jet production where, due to the high center-of-mass energy at LHC, large statistics is also expected. At the same time, the process $t \bar{t}+$ jets is an important background to Higgs or supersymmetry searches, so that experimental search strategies need to impose kinematical cuts on the final state. At tree level, the cross sections have a really large scale dependence, which is why NLO QCD corrections are mandatory. For instance, the process $t \bar{t}+2$ jets entered table 2 as a specific background to $t \bar{t} H$, where the Higgs decays into a $b \bar{b}$-pair. The NLO QCD corrections to the former would help to control the background uncertainty due to a heavy flavor mistag in a $t \bar{t}+2$ jets event.

On the way to this challenge (six-leg processes being currently at the edge of technology), the process $t \bar{t}+1$ jet was computed recently to NLO QCD in an impressive state-of-the-art calculation [144]. Figure 17 displays the much improved scale dependence and shows that the perturbative corrections are moderate for the nominal scale choice $\mu \simeq m_{t}$. Clearly, it will be very interesting to see the NLO differential distributions for this reaction in the future and to compare them with LO predictions (e.g. from MadGraph) combined with parton showers. In this way, one can assess how well the NLO predictions for jet observables are modeled by the underlying partonic processes such as $g g \rightarrow t \bar{t} g$.

\section{Higgs production at LHC}

Let us conclude this review with a brief discussion of the flagship measurement to be conducted at LHC. To start with, we illustrate in figure 18 for the Standard Model Higgs the dominant production modes (left) and the branching ratios for the decay (right) as a function of the Higgs mass. The plotted values for the mass range up to $M_{H}=1 \mathrm{TeV}$, which is generally 

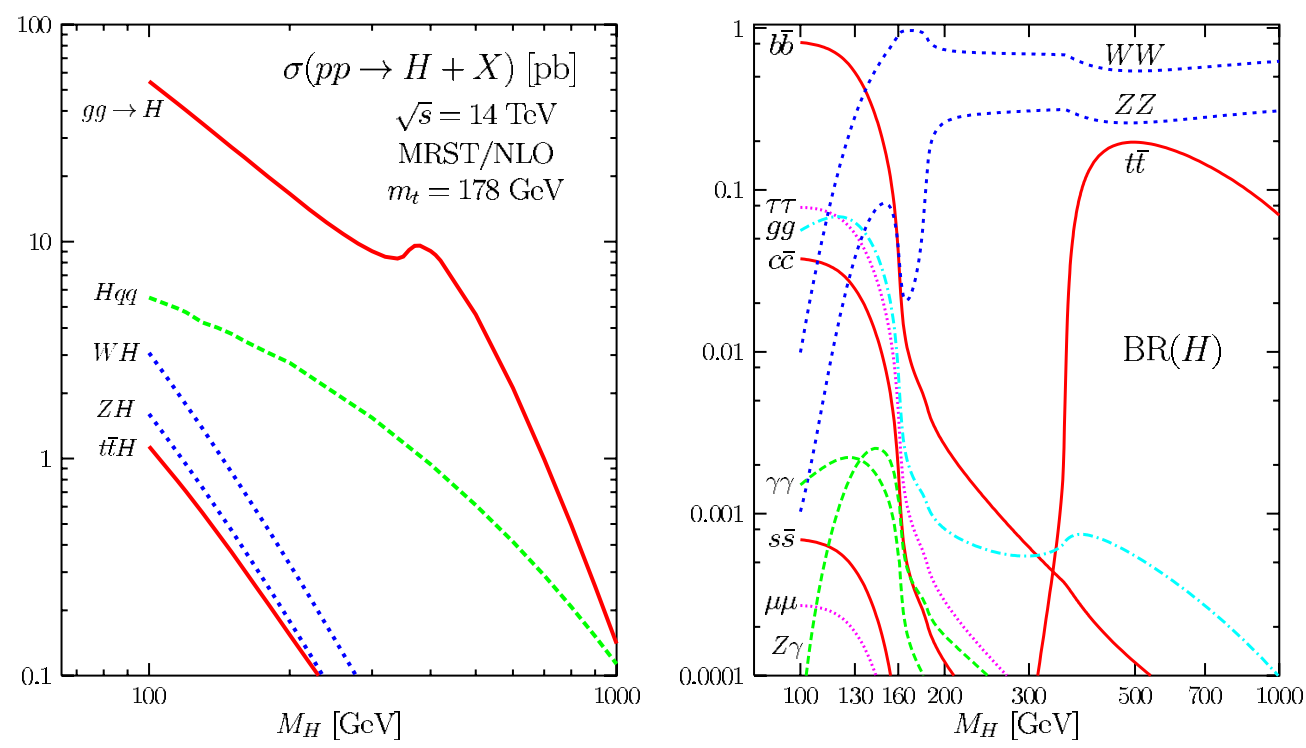

Figure 18. Left: the total cross section for Higgs production at LHC at NLO in QCD. Right: the branching ratios of Higgs boson decay (Reprinted from 2008 Phys. Rep. 4571 with permission from Elsevier).
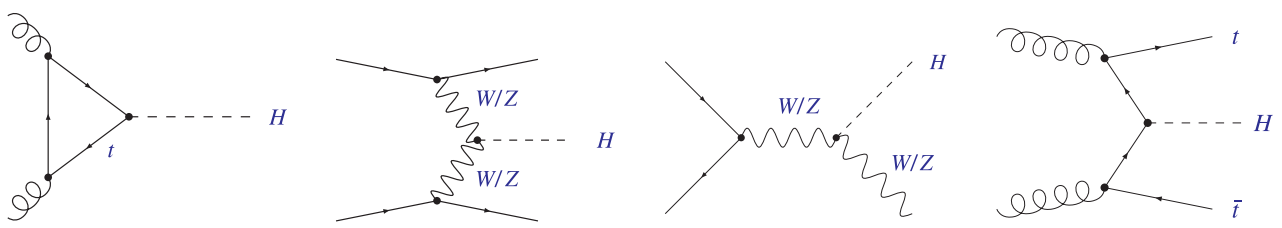

Figure 19. Sample of Feynman diagrams for the various modes of Higgs production. Gluon fusion (left), weak vector-boson fusion (second from left), Higgs-strahlung (second from right) and $t \bar{t}$ H-channel (right).

considered an upper bound for the Standard Model Higgs due to triviality. A lower bound on the Higgs mass has been established from direct searches at LEP [145, 146], currently $M_{H}=114.4 \mathrm{GeV}$. With the high luminosity and statistics of run-II the Tevatron experiments currently conduct an active search for the Higgs as well (see [147]).

Focusing on hard QCD aspects, we will limit ourselves to the production part (i.e. figure 18 on the left). Depending on the Higgs mass, the signatures from the various decay modes (gauge bosons, lepton pairs, quark pairs, etc) define the experimental search strategy and, at the same time of course, the need to improve predictions for the competing Standard Model processes as discussed in section 4. Unfortunately, this will not always be successful, as for example the fully hadronic modes will not be accessible for Higgs detection because of the huge QCD multi-jet background. However, due to the numerous channels we can hardly touch these aspects. A very extensive discussion of Higgs physics at colliders can be found, e.g., in the recent $[148,149]$.

As can seen from figure 18 on the left, we have a clear hierarchy of channels and in figure 19 we display samples of Feynman diagrams for these production modes. Gluon fusion is induced via a heavy-quark loop (figure 19 left). It has the largest rate for all values of the Higgs mass $M_{H}$ due to the large top-Yukawa coupling of the Higgs boson and the large 
gluon luminosity. In limit of a heavy top $\left(m_{t} \rightarrow \infty\right)$ one can describe the interaction by an effective $g g H$-vertex upon integrating out the heavy quark in the loop, which is also a very good approximation for finite $m_{t}$. QCD corrections for numerous observables in this channel have been determined and we will highlight a few aspects below.

Weak vector-boson fusion proceeds via $q q \rightarrow q q H$ (figure 19 second from left) and is mediated by $t$-channel gauge-boson exchange. It has the second largest rate, being dominated mostly by the $u, d$-quark PDFs and is proportional to the $W W H$ coupling. However, the signal identification for the three-body final state needs dedicated cuts on the final state jets (see, e.g., [150, 151]). The characteristics of the latter are extremely important to discriminate the VBF signal from QCD backgrounds, for instance through forward jet-tagging and central jet-vetoing (see, e.g., [152]). Higgs-strahlung in the channel $q \bar{q} \rightarrow W(Z) H$ (figure 19 second from right) makes up for the third largest rate and has the same couplings as vector-boson fusion. The production mechanism requires a gauge boson from $q \bar{q}$-annihilation so that the radiative corrections in QCD for Higgs-strahlung follow largely from the corresponding ones in the Drell-Yan process and have been determined to NNLO [153].

As another mode the associated Higgs production with heavy quarks has been discussed, for example $p p \rightarrow t \bar{t} H$ (figure 19 right), which is now known to NLO in QCD [154-157]. At LHC the process is driven by the gluon luminosity but the rate drops quickly for larger Higgs masses and the phase space becomes too small already for values of $M_{H} \simeq 180 \mathrm{GeV}$. In addition, for lower Higgs masses the final state from $p p \rightarrow t \bar{t} H$ has a large Standard Model background (see table 2) which will be difficult to suppress. The process $p p \rightarrow b \bar{b} H$ even has a slightly larger rate at LHC for $M_{H} \leqslant 300 \mathrm{GeV}$, but the final state with $b$-jets is overwhelmed by background. Generally, Higgs couplings to bottom quarks are more important in the extended Higgs sector of the MSSM (see, e.g., [149]). Finally, the production of Higgs boson pairs $(p p \rightarrow H H+X)$ at LHC still has rates accessible to measurements for low Higgs masses, however we will not discuss these processes further. Cross sections for the production of three or more Higgs bosons at LHC are too small [148].

Let us, for the rest of this section, focus on the role of higher order QCD corrections for the Higgs signal in gluon fusion. The total cross section for gluon fusion in the heavy top limit is known exactly to the NNLO in QCD [158-160]. The radiative corrections yield a sizable $K$-factor of two or more as can be seen in figure 20 (left) where we display the total cross section as a function of $M_{H}$ in the low mass range. Of course, the origin of this large effect is well understood. It is due to soft gluon emission, which makes up numerically for the bulk of the perturbative corrections (see, e.g., [161] and references therein). This fact has motivated the derivation of the complete soft $\mathrm{N}^{3} \mathrm{LO}$ corrections in [161] which are also plotted in figure 20 and nicely illustrate the property of apparent convergence of the perturbative expansion. Another indicator in this respect is, as often stressed so far, the stability under scale variation in a typical range, say $M_{H} \leqslant \mu \leqslant 2 M_{H}$. This is shown in figure 20 (right) where we display the total cross section as a function of the renormalization scale for a Higgs mass $M_{H}=120 \mathrm{GeV}$. As the scale-dependent terms are completely predicted by the lower order terms, the curve denoted by $\mathrm{N}^{3} \mathrm{LO}_{\text {approx }}$ should be a very good approximation of the exact three-loop result with a residual uncertainty estimated of $\mathcal{O}(1-2 \%)$ only.

Comparison with experimental data of course requires selection cuts on differential distributions. The latter are available for the gluon fusion channel including QCD predictions up to NNLO and allow us to study bin-integrated distributions (e.g. for the Higgs rapidity) with subsequent Higgs decay in a variety of modes. Presently, the NNLO corrections in gluon fusion have been combined with the decay modes $H \rightarrow \gamma \gamma$ as well as $H \rightarrow W W \rightarrow l v l v$ and $H \rightarrow Z Z \rightarrow 4 l[28,162,29,163]$. It is very interesting to study these higher order corrections under the impact of kinematical cuts on the observed final state leptons, photons 

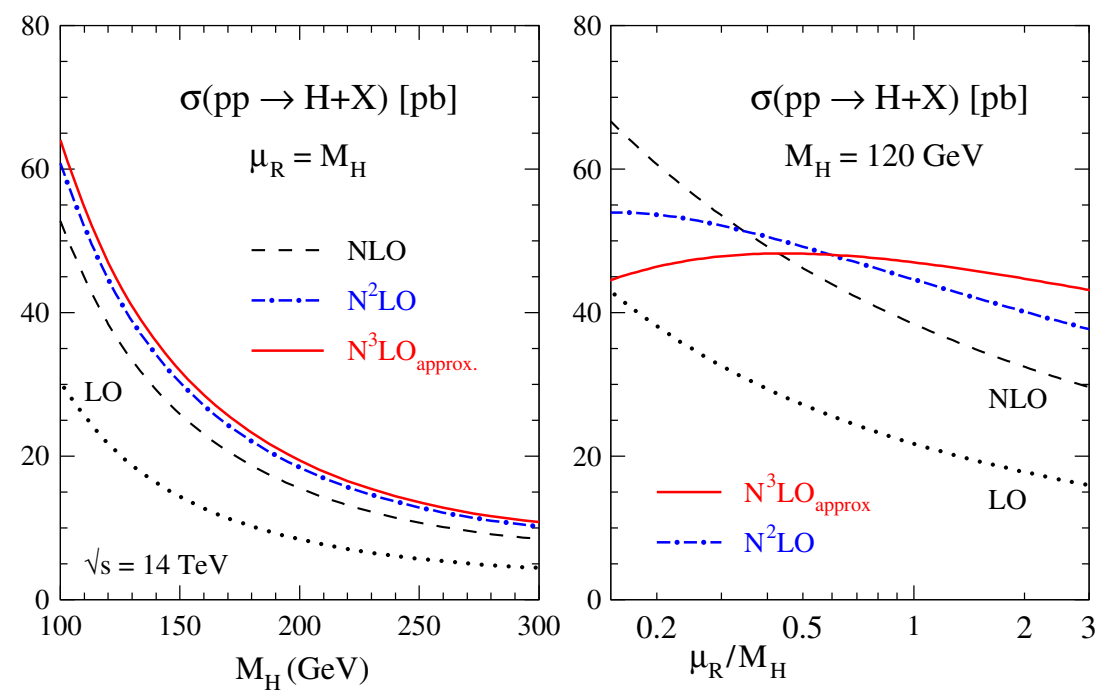

Figure 20. Perturbative expansion of the total cross section for Higgs production at the LHC. Shown are the dependence on the mass $M_{H}$ and the renormalization scale $\mu_{\mathrm{r}}$ (Reprinted from 2005 Phys. Lett. B 63148 with permission from Elsevier).
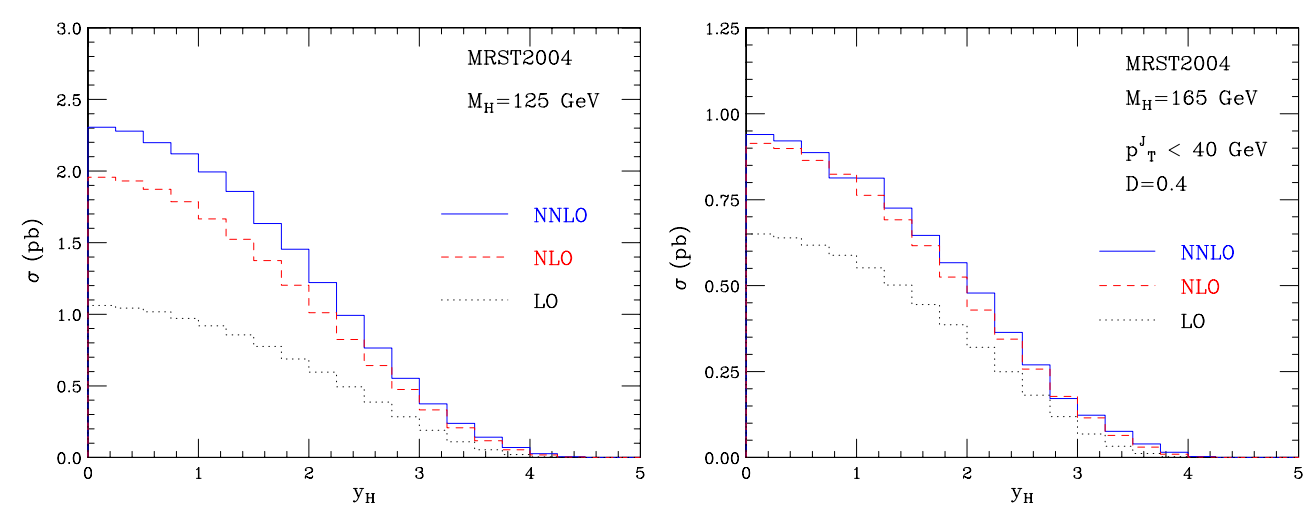

Figure 21. Left: Higgs mass $M_{h}=125 \mathrm{GeV}$, no cuts on $p_{t}$ of jets. Right: Higgs mass $M_{h}=$ $165 \mathrm{GeV}$ and veto on jets with $p_{t}>40 \mathrm{GeV}\left(k_{t}\right.$ algorithm for jet reconstruction with jet size $D=0.4$ ) (Reprinted with permission from 2007 Phys. Rev. Lett. 98222002 . Copyright (2007) by the American Physical Society).

or the jet activity. Contrary to the findings for the total cross section, where higher order corrections amount to $100 \%$ or more, the effect of radiative corrections in distributions is strongly reduced by the selection cuts. We illustrate this for the Higgs rapidity distribution as calculated with the parton level Monte Carlo program HNNLO [29]. In figure 21, on the left no cuts on the $p_{t}$ of additional jets are applied and the increase of the NNLO over the NLO corrections amounts to approximately $20 \%$ with some dependence on the rapidity. In contrast, in figure 21 on the right all jets with $p_{t} \geqslant 40 \mathrm{GeV}$ have been vetoed, a situation typical when searching for the Higgs in the decay mode $H \rightarrow W W$ to suppress the $W W$ background from $t \bar{t}$-production. As an upshot, the size of NNLO QCD radiative corrections is reduced to $5 \%$. Moreover, as expected, the NNLO QCD corrections significantly improve the stability under 
scale variation. First steps toward an assessment of the $W W$-background from QCD at NNLO have recently been made [164]. Also we remark that for the Higgs rapidity distribution even the soft $\mathrm{N}^{3} \mathrm{LO}$ corrections have been obtained and simple analytical formulae are available [165].

In summary, we conclude that the rates for the Standard Model Higgs at LHC are reliably predicted by QCD. We have illustrated this for the gluon fusion channel where we have observed how higher order QCD predictions decrease the sensitivity to scale variations. There exists a residual uncertainty of the cross section of a few per cent due to the parton luminosity (see section 3.4). However, the gluon PDF is well constrained in the kinematical range and HERA data can be evolved to NNLO accuracy. The study of differential distributions in particular with realistic experimental cuts is an active field of ongoing research for all main production modes (see figure 19) and we refer to the literature (see, e.g., [7, 8, 62, 148]).

\section{Summary}

We have briefly reviewed the theoretical framework of QCD at hadron colliders. Precision predictions for hard-scattering cross sections rely on a detailed knowledge of the parton content of the proton and of the rates for the corresponding partonic subprocess. We have given an overview of our current information on parton distributions including evolution to LHC energies. For various Standard Model cross sections such as $W^{ \pm} / Z$-boson, Higgs boson or top quark production we have reported on the present status of perturbative QCD predictions. Precision cross sections require the calculation of higher order corrections for both, the signal and the background with massive particles and jets. A lot of ongoing activities in this direction are concentrated on processes with multi-particle production and we have tried to give a snapshot of the technology involved, e.g. the idea on shell recursions for scattering amplitudes. In summary, we have tried to convey the message that QCD theory is ready to meet the challenges of LHC.

In this review, we have mostly omitted details of specific hadronic final states, e.g. jet algorithms, $b$-quark ( $b$-jet) production or aspects of $b$-quark fragmentation as well as parton showers in Monte Carlo simulations. We have also left out any discussion of resummation approaches meant to improve fixed order perturbation theory, be it threshold logarithms of Sudakov type or $\ln \left(p_{t}\right)$-terms in transverse momentum. Finally, nothing has been said about the region of small- $x$ and forward physics at large rapidities (e.g. for diffractive production of Higgs bosons). For all these remaining aspects as well as a broader coverage, the interested reader is referred to $[2,3,7,8,37,38,62]$ and the numerous references therein.

QCD theory at hadron colliders is an extremely dynamical field at the moment, thus we expect that many specific issues will be improved or further clarified soon. The broad theoretical framework, however, will certainly remain valid and, hopefully, the present compilation has highlighted the role of QCD in the era of LHC.

\section{Acknowledgments}

Feynman diagrams have been drawn with the packages AXODRAW [166] and JAXO-DRAW [167]. The work has been supported in part by the Helmholtz Gemeinschaft under contract VH-NG105 .

\section{References}

[1] ATLAS, CERN-LHCC-1999-014 ATLAS-TDR-014 1999 Available at http://cdsweb.cern.ch/

[2] ATLAS, CERN-LHCC-1999-015, ATLAS-TDR-015 1999 Available at http://cdsweb.cern.ch/ 
[3] CMS, CERN-LHCC-2006-021, CMS-TDR-008-2 2006 Available at http://cdsweb.cern.ch/

[4] Bayatian G L et al (CMS) 2007 J. Phys. G: Nucl. Part. Phys. 34995

[5] LHCb, CERN-LHCC-2003-030, LHCb TDR 9 2003, Available at http://cdsweb.cern.ch/

[6] ALICE, CERN-LHCC-2005-030, ALICE-TDR-13 2005 Available at http://cdsweb.cern.ch/

[7] Campbell J M, Huston J W and Stirling W J 2007 Rep. Prog. Phys. 7089 (Preprint hep-ph/0611148)

[8] Dixon L J 2007 Preprint arXiv:0712.3064

[9] Gianotti F and Mangano M L 2005 Preprint hep-ph/0504221

[10] Collins J C, Soper D E and Sterman G 1988 Adv. Ser. Direct. High Energy Phys. 51 (Preprint hep-ph/0409313)

[11] Corcella G et al 2002 Preprint hep-ph/0210213

[12] Sjostrand T, Mrenna S and Skands P 2006 J. High Energy Phys. JHEP05(2006)026 (Preprint hep-ph/0603175)

[13] Sjostrand T, Mrenna S and Skands P 2007 Preprint arXiv:0710.3820

[14] Gleisberg T et al 2004 J. High Energy Phys. JHEP02(2004)056 (Preprint hep-ph/0311263)

[15] Mangano M L et al 2003 J. High Energy Phys. JHEP07(2003)001 (Preprint hep-ph/0206293)

[16] Boos E et al (CompHEP) 2004 Nucl. Instrum. Methods A 534250 (Preprint hep-ph/0403113)

[17] Cafarella A, Papadopoulos C G and Worek M 2007 Preprint arXiv:0710.2427

[18] Maltoni F and Stelzer T 2003 J. High Energy Phys. JHEP02(2003)027 (Preprint hep-ph/0208156)

[19] Kilian W, Ohl T and Reuter J 2007 Preprint arXiv:0708.4233

[20] Nagy Z 2002 Phys. Rev. Lett. 88122003 (Preprint hep-ph/0110315)

[21] Nagy Z 2003 Phys. Rev. D 68094002 (Preprint hep-ph/0307268)

[22] Campbell J M and Ellis R K 2000 Phys. Rev. D 62114012 (Preprint hep-ph/0006304)

[23] Frixione S, Nason P and Webber B R 2003 J. High Energy Phys. JHEP08(2003)007 (Preprint hep-ph/0305252)

[24] Frixione S and Webber B R 2006 Preprint hep-ph/0612272

[25] Nason P 2004 J. High Energy Phys. JHEP11(2004)040 (Preprint hep-ph/0409146)

[26] Frixione S, Nason P and Oleari C 2007 J. High Energy Phys. JHEP11(2007)070 (Preprint 0709.2092)

[27] Giele W T, Kosower D A and Skands P Z 2007 Preprint arXiv:0707.3652

[28] Anastasiou C, Melnikov K and Petriello F 2005 Nucl. Phys. B 724197 (Preprint hep-ph/0501130)

[29] Catani S and Grazzini M 2007 Phys. Rev. Lett. 98222002 (Preprint hep-ph/0703012)

[30] Vermaseren J A M, Vogt A and Moch S 2005 Nucl. Phys. B 7243 (Preprint hep-ph/0504242)

[31] Collins J C and Soper D E 1982 Nucl. Phys. B 194445

[32] Moch S, Vermaseren J A M and Vogt A 2004 Nucl. Phys. B 688101 (Preprint hep-ph/0403192)

[33] Vogt A, Moch S and Vermaseren J A M 2004 Nucl. Phys. B 691129 (Preprint hep-ph/0404111)

[34] Vogt A 2005 Comput. Phys. Commun. 17065 (Preprint hep-ph/0408244)

[35] Salam G http://projects.hepforge.org/hoppet/

[36] Botje M http://www.nikhef.nl/h24/qcdnum/

[37] Giele W et al 2002 Preprint hep-ph/0204316

[38] Dittmar M et al 2005 Preprint hep-ph/0511119

[39] Martin A D et al 2004 Phys. Lett. B 60461 (Preprint hep-ph/0410230)

[40] Whalley M R, Bourilkov D and Group R C 2005 Preprint hep-ph/0508110

[41] CEDAR HepForge 2007 http://projects.hepforge.org/lhapdf/

[42] Yao W M et al (Particle Data Group) 2006 J. Phys. G: Nucl. Part. Phys. 331

[43] Vogt A 2007 Preprint arXiv:0707.4106

[44] Chekanov S et al (ZEUS) 2003 Phys. Rev. D 67012007 (Preprint hep-ex/0208023)

[45] Dittmar M, Pauss F and Zurcher D 1997 Phys. Rev. D 567284 (Preprint hep-ex/9705004)

[46] Martin A D et al 2002 Phys. Lett. B 531216 (Preprint hep-ph/0201127)

[47] Anastasiou C et al 2004 Phys. Rev. D 69094008 (Preprint hep-ph/0312266)

[48] Nadolsky P M et al 2008 Preprint arXiv:0802.0007

[49] Pumplin J et al 2002 J. High Energy Phys. JHEP07(2002)012 (Preprint hep-ph/0201195)

[50] Thorne R S et al 2007 Preprint arXiv:0706.0456

[51] Martin A D et al 2007 Phys. Lett. B 652292 (Preprint 0706.0459)

[52] Chuvakin A, Smith J and van Neerven W L 2000 Phys. Rev. D 61096004 (Preprint hep-ph/9910250)

[53] Tung W K, Kretzer S and Schmidt C 2002 J. Phys. G: Nucl. Part. Phys. 28983 (Preprint hep-ph/0110247)

[54] Anchordoqui L A et al 2007 Preprint arXiv:0712.0386

[55] Ferrag S (ATLAS) 2004 Preprint hep-ph/0407303

[56] Pumplin J, Lai H L and Tung W K 2007 Phys. Rev. D 75054029 (Preprint hep-ph/0701220)

[57] Dittmaier S, Kallweit S and Uwer P 2007 Preprint arXiv:0710.1577

[58] Campbell J M, Ellis R K and Zanderighi G 2007 Preprint arXiv:0710.1832

[59] Campbell J M, Ellis R K and Zanderighi G 2006 J. High Energy Phys. JHEP10(2006)028 (Preprint hep-ph/0608194) 
[60] Lazopoulos A, Melnikov K and Petriello F 2007 Phys. Rev. D 76014001 (Preprint hep-ph/0703273)

[61] Hankele V and Zeppenfeld D 2008 Preprint arXiv:0712.3544

[62] Buttar C et al 2006 Preprint hep-ph/0604120

[63] Passarino G and Veltman M 1979 Nucl. Phys. B 160151

[64] Binoth T, Guillet J P and Heinrich G 2000 Nucl. Phys. B 572361 (Preprint hep-ph/9911342)

[65] Denner A and Dittmaier S 2006 Nucl. Phys. B 73462 (Preprint hep-ph/0509141)

[66] Ossola G, Papadopoulos C G and Pittau R 2007 Nucl. Phys. B 763147 (Preprint hep-ph/0609007)

[67] Hahn T 2000 Nucl. Phys. Proc. Suppl. 89231 (Preprint hep-ph/0005029)

[68] Ellis R K, Stirling W J and Webber B R 2003 QCD and Collider Physics (Cambridge: Cambridge University Press) ISBN 0-521-58189-3

[69] Catani S and Seymour M H 1997 Nucl. Phys. B 485291 (Preprint hep-ph/9605323)

[70] Catani S et al 2002 Nucl. Phys. B 627189 (Preprint hep-ph/0201036)

[71] Bern Z, Dixon L J and Kosower D A 2007 Ann. Phys. 3221587 (Preprint 0704.2798)

[72] Berends F A and Giele W T 1988 Nucl. Phys. B 306759

[73] Dixon L J 1996 Preprint hep-ph/9601359

[74] Parke S J and Taylor T R 1986 Phys. Rev. Lett. 562459

[75] Mangano M L and Parke S J 1991 Phys. Rep. 200301 (Preprint hep-th/0509223)

[76] Witten E 2004 Commun. Math. Phys. 252189 (Preprint hep-th/0312171)

[77] Britto R, Cachazo F and Feng B 2005 Nucl. Phys. B 715499 (Preprint hep-th/0412308)

[78] Britto R et al 2005 Phys. Rev. Lett. 94181602 (Preprint hep-th/0501052)

[79] Dixon L J, Glover E W N and Khoze V V 2004 J. High Energy Phys. JHEP12(2004)015 (Preprint hep-th/0411092)

[80] Badger S D, Glover E W N and Khoze V V 2005 J. High Energy Phys. JHEP03(2005)023 (Preprint hep-th/0412275)

[81] Mastrolia P 2006 Nucl. Phys. Proc. Suppl. 160209 (Preprint hep-ph/0607011)

[82] Bern Z et al 1995 Nucl. Phys. B 43559 (Preprint hep-ph/9409265)

[83] Britto R, Cachazo F and Feng B 2005 Nucl. Phys. B 725275 (Preprint hep-th/0412103)

[84] Bern Z et al 1994 Nucl. Phys. B 425217 (Preprint hep-ph/9403226)

[85] Bern Z, Dixon L J and Kosower D A 1995 Nucl. Phys. B 437259 (Preprint hep-ph/9409393)

[86] Bidder S J et al 2005 Phys. Lett. B 606189 (Preprint hep-th/0410296)

[87] Bedford J et al 2005 Nucl. Phys. B 71259 (Preprint hep-th/0412108)

[88] Bidder S J et al 2005 Phys. Lett. B 61275 (Preprint hep-th/0502028)

[89] Britto R et al 2005 Phys. Rev. D 72065012 (Preprint hep-ph/0503132)

[90] Bern Z, Dixon L J and Kosower D A 2006 Phys. Rev. D 73065013 (Preprint hep-ph/0507005)

[91] Bern Z et al 2005 J. High Energy Phys. JHEP11(2005)027 (Preprint hep-ph/0507019)

[92] Britto R, Feng B and Mastrolia P 2006 Phys. Rev. D 73105004 (Preprint hep-ph/0602178)

[93] Berger C F et al 2006 Phys. Rev. D 74036009 (Preprint hep-ph/0604195)

[94] Berger C F et al 2007 Phys. Rev. D 75016006 (Preprint hep-ph/0607014)

[95] Xiao Z, Yang G and Zhu C J 2006 Nucl. Phys. B 75853 (Preprint hep-ph/0607017)

[96] Ellis R K, Giele W T and Zanderighi G 2006 J. High Energy Phys. JHEP05(2006)027 (Preprint hep-ph/0602185)

[97] Giele W T, Kunszt Z and Melnikov K 2008 Preprint arXiv:0801.2237

[98] Schwinn C and Weinzierl S 2007 J. High Energy Phys. JHEP04(2007)072 (Preprint hep-ph/0703021)

[99] Britto R and Feng B 2007 Phys. Rev. D 75105006 (Preprint hep-ph/0612089)

[100] Ciccolini M, Denner A and Dittmaier S 2007 Phys. Rev. Lett. 99161803 (Preprint 0707.0381)

[101] Figy T, Hankele V and Zeppenfeld D 2007 Preprint arXiv:0710.5621

[102] Jäger B, Oleari C and Zeppenfeld D 2006 J. High Energy Phys. JHEP07(2006)015 (Preprint hep-ph/0603177)

[103] Jäger B, Oleari C and Zeppenfeld D 2006 Phys. Rev. D 73113006 (Preprint hep-ph/0604200)

[104] Bozzi G et al 2007 Phys. Rev. D 75073004 (Preprint hep-ph/0701105)

[105] CEDAR HepCode 2007 http://www.cedar.ac.uk/hepcode/

[106] Binoth T et al 2000 Eur. Phys. J. C 16311 (Preprint hep-ph/9911340)

[107] Aurenche P et al 2006 Phys. Rev. D 73094007 (Preprint hep-ph/0602133)

[108] Balazs C et al 1998 Phys. Rev. D 576934 (Preprint hep-ph/9712471)

[109] Balazs C et al 2007 Phys. Rev. D 76013009 (Preprint arXiv:0704.0001)

[110] Balazs C and Yuan C P 1997 Phys. Rev. D 565558 (Preprint hep-ph/9704258)

[111] Denner A et al 2005 Phys. Lett. B 612223 (Preprint hep-ph/0502063)

[112] Denner A et al 2005 Nucl. Phys. B 724247 (Preprint hep-ph/0505042)

[113] Belanger G et al 2003 Nucl. Phys. Proc. Suppl. 116353 (Preprint hep-ph/0211268) 
[114] Boudjema F et al 2005 Preprint hep-ph/0510184

[115] Lei G et al 2007 Preprint arXiv:0708.2951

[116] Gehrmann-De Ridder A et al 2007 Phys. Rev. Lett. 99132002 (Preprint 0707.1285)

[117] Gehrmann-De Ridder A et al 2007 J. High Energy Phys. JHEP12(2007)094 (Preprint 0711.4711)

[118] Quadt A 2006 Eur. Phys. J. C 48835

[119] The Tevatron Electroweak Working Group (TEV EWWG) for CDF and DZero 2007 Preprint hep-ex/0703034

[120] Frederix R and Maltoni F 2007 Preprint arXiv:0712.2355

[121] Nason P, Dawson S and Ellis R K 1988 Nucl. Phys. B 303607

[122] Beenakker W et al 1989 Phys. Rev. D 4054

[123] CDF 2006 CDF-note-8148 Available at http://www-cdf.fnal.gov

[124] Tung W K et al 2007 J. High Energy Phys. JHEP02(2007)053 (Preprint hep-ph/0611254)

[125] Moch S and Uwer P 2008 Preprint arXiv:0804.1476

[126] Kidonakis N and Sterman G 1997 Nucl. Phys. B 505321 (Preprint hep-ph/9705234)

[127] Bonciani R et al 1998 Nucl. Phys. B 529424 (Preprint hep-ph/9801375)

[128] Kidonakis N et al 2001 Phys. Rev. D 64114001 (Preprint hep-ph/0105041)

[129] Körner J G, Merebashvili Z and Rogal M 2008 Preprint arXiv:0802.0106

[130] Czakon M, Mitov A and Moch S 2007 Phys. Lett. B 651147 (Preprint arXiv:0705.1975)

[131] Czakon M, Mitov A and Moch S 2007 Preprint arXiv:0707.4139

[132] Mitov A and Moch S 2007 J. High Energy Phys. JHEP05(2007)001 (Preprint hep-ph/0612149)

[133] Moch S and Mitov A 2007 Acta Phys. Polon. B 383507 (Preprint arXiv:0711.1121)

[134] Melnikov K and Mitov A 2004 Phys. Rev. D 70034027 (Preprint hep-ph/0404143)

[135] Mitov A 2005 Phys. Rev. D 71054021 (Preprint hep-ph/0410205)

[136] Mitov A, Moch S and Vogt A 2006 Phys. Lett. B 63861 (Preprint hep-ph/0604053)

[137] Moch S and Vogt A 2008 Phys. Lett. B 659290 (Preprint arXiv:0709.3899)

[138] Kühn J H, Scharf A and Uwer P 2007 Eur. Phys. J. C 5137 (Preprint hep-ph/0610335)

[139] Hooper D and Profumo S 2007 Phys. Rep. 45329 (Preprint hep-ph/0701197)

[140] Abazov V M et al (D0) 2007 Phys. Rev. Lett. 98181802 (Preprint hep-ex/0612052)

[141] Mitrevski J (f.t. CDF and D. Collaborations) 2008 Preprint arXiv:0802.2965

[142] Sullivan Z 2004 Phys. Rev. D 70114012 (Preprint hep-ph/0408049)

[143] Frixione S et al 2006 J. High Energy Phys. JHEP03(2006)092 (Preprint hep-ph/0512250)

[144] Dittmaier S, Uwer P and Weinzierl S 2007 Phys. Rev. Lett. 98262002 (Preprint hep-ph/0703120)

[145] Barate R et al (LEP Working Group for Higgs Boson Searches) 2003 Phys. Lett. B 56561 (Preprint hep-ex/0306033)

[146] The LEP Electroweak Working Group (LEP EWWG) 2007 http://lepewwg.web.cern.ch/LEPEWWG/

[147] The Tevatron Electroweak Working Group (TEV EWWG) 2007 http://tevewwg.fnal.gov/

[148] Djouadi A 2008 Phys. Rep. 4571 (Preprint hep-ph/0503172)

[149] Djouadi A 2005 Preprint hep-ph/0503173

[150] Rainwater D L and Zeppenfeld D 1997 J. High Energy Phys. JHEP12(1997)005 (Preprint hep-ph/9712271)

[151] Rainwater D L and Zeppenfeld D 1999 Phys. Rev. D 60113004 (Preprint hep-ph/9906218)

[152] Rainwater D L 1999 Preprint hep-ph/9908378

[153] Brein O, Djouadi A and Harlander R 2004 Phys. Lett. B 579149 (Preprint hep-ph/0307206)

[154] Beenakker W et al 2001 Phys. Rev. Lett. 87201805 (Preprint hep-ph/0107081)

[155] Beenakker W et al 2003 Nucl. Phys. B 653151 (Preprint hep-ph/0211352)

[156] Dawson S et al 2003 Phys. Rev. D 67071503 (Preprint hep-ph/0211438)

[157] Dawson S et al 2003 Phys. Rev. D 68034022 (Preprint hep-ph/0305087)

[158] Harlander R V and Kilgore W B 2002 Phys. Rev. Lett. 88201801 (Preprint hep-ph/0201206)

[159] Anastasiou C and Melnikov K 2002 Nucl. Phys. B 646220 (Preprint hep-ph/0207004)

[160] Ravindran V, Smith J and van Neerven W L 2003 Nucl. Phys. B 665325 (Preprint hep-ph/0302135)

[161] Moch S and Vogt A 2005 Phys. Lett. B 63148 (Preprint hep-ph/0508265)

[162] Anastasiou C, Dissertori G and Stockli F 2007 J. High Energy Phys. JHEP09(2007)018 (Preprint hep-ph/0707.2373)

[163] Grazzini M 2008 Preprint arXiv:0801.3232

[164] Chachamis G, Czakon M and Eiras D 2008 Preprint arXiv:0802.4028

[165] Ravindran V, Smith J and van Neerven W L 2007 Nucl. Phys. B 767100 (Preprint hep-ph/0608308)

[166] Vermaseren J A M 1994 Comput. Phys. Commun. 8345

[167] Binosi D and Theussl L 2004 Comput. Phys. Commun. 16176 (Preprint hep-ph/0309015) 